OPEN ACCESS

Edited by:

Dejian Huang,

National University of

Singapore, Singapore

Reviewed by:

Sui Kiat Chang

South China Botanical Garden,

Chinese Academy of Sciences, China

Marcin Szymanski,

Adam Mickiewicz University, Poland

*Correspondence:

Shiyi Ou

tosy@jnu.edu.cn orcid.org/0000-0002-6779-0858

Pengzhan Liu

Ipzhan@scut.edu.cn

orcid.org/0000-0003-2703-1993

tThese authors have contributed equally to this work

Specialty section:

This article was submitted to

Food Chemistry

a section of the journal

Frontiers in Nutrition

Received: 10 June 2020

Accepted: 26 August 2020

Published: 09 November 2020

Citation:

Zheng J, Wu Z, Yang N, Zhou K,

Hu W, Ou S and Liu P (2020) Widely

Targeted UHPLC-MS/MS

Metabolomic Analysis on the

Chemical Variation in Blueberry-Filled

Pastries During Processing.

Front. Nutr. 7:569172.

doi: 10.3389/fnut.2020.569172

\section{Widely Targeted UHPLC-MS/MS Metabolomic Analysis on the Chemical Variation in Blueberry-Filled Pastries During Processing}

\author{
Jie Zheng ${ }^{1 \dagger}$, Zhongjun $\mathrm{Wu}^{1+}{ }^{1+}$, Nan Yang ${ }^{1}$, Kangning Zhou ${ }^{1}$, Wenzhong $\mathrm{Hu}^{2,3}$, Shiyi Ou ${ }^{1 *}$ and \\ Pengzhan Liu ${ }^{4 *}$
}

\begin{abstract}
${ }^{1}$ Department of Food Science and Engineering, Jinan University, Guangzhou, China, ${ }^{2}$ College of Life Science, Dalian Minzu University, Dalian, China, ${ }^{3}$ Key Laboratory of Biotechnology and Bioresources Utilization, Dalian Minzu University, Dalian,

China, ${ }^{4}$ School of Food Science and Engineering, South China University of Technology, Guangzhou, China
\end{abstract}

The majority of components in fruits are sensitive to heat-processing. Nevertheless, fruits are becoming popular ingredients in processed foods, like bakery foods. Therefore, the fate of the components in the fruit-involved food during thermal processing is important for the assessment of their nutritional values and sensory properties. Unfortunately, comprehensive knowledge of the compositional alteration in real food products during processing is limited. In the current study, a popular bakery food, blueberry-filled pastry, was taken as the object, and a widely targeted metabolomic approach was applied to investigate the holistic compositional variation of blueberry filling during pastry preparation. Amongst the total of 630 chemicals identified, 288 chemicals were screened as differential compounds between samples collected at different processing stages. The most variation of the chemicals was observed during the process of stir-frying. A total of 197 chemicals varied significantly in concentrations during stir-frying, while only 75 chemicals altered significantly in contents during baking. Amongst 288 differential compounds, 117 belonged to the group of phenolic compounds, with the others found to be sugars and organic acids, amino acids, lipids, nucleotides, etc. The possible mechanisms of the chemical alterations during thermal processing were also discussed in the current study. The data provide comprehensive information on the compositional changes in berry-containing fillings during thermal processing, and hints and emphasis for further investigation of the underlying mechanisms.

Keywords: baking, stir-frying, metabolomic analysis, chemical variation, degradation

\section{INTRODUCTION}

Blueberries contain various phytochemicals with potential health effects, including anthocyanins, flavonols, vitamins, lutein, sugars, and organic acid (1-3). The consumption of polyphenol-rich blueberries and their polyphenol extracts is associated with the protection and management of non-communicable diseases such as cancers, 
cardiovascular diseases, diabetes, osteoporosis, and neurodegenerative diseases (1). However, the perishable nature and seasonal availability of berries limit the all-yearround supply and consumption. Food processing allows the all-year-round provision and provides various types of blueberry products to compensate for the different requirements and appreciations by the customers. One large group of products are bakery food products filled with blueberries or blueberry containing materials, such as pies, pastries, muffins, cakes, and cookies. Such food products are becoming increasingly popular and are consumed daily in many areas of the world. Processing can prolong the shelf life of blueberry products and increase the diversity of products for customers' choice; however, nutritional compounds, such as polyphenols, may be destroyed to some extent during processing, especially during thermal processing, which in turn affects the health-promoting properties of the products (4-6).

However, knowledge of the effect of thermal processing on the phytochemical profile of the products is limited to a few compounds due to the conventional analytical methods focusing only on a certain group of chemicals $(3,7,8)$, although the biological importance is determined by the whole phytochemical profile. Thus, a technique should be developed to fully elucidate the components in a food product for evaluating the nutritional changes during thermal processing. Metabolomic analysis is used to provide a comprehensive chemical profile of as many small molecules as possible in a system (plant, cell, or food material), which allows for a more thorough and encompassing analysis of molecular composition than traditional methods. The application of metabolomic study on the tracking of dynamic compositional changes during food processing could provide a holistic view on the chemical changes and correlated reactions under various treatments of food. Thus, in recent years, metabolomic analysis has been applied in food products, such as in the investigation of nectar beverage of black raspberries (9), roasted coffee beans (10), wines (11), and extra-virgin olive oil (12).

In our previous study (8), the variation in individual flavonoids, including flavonol glycosides and anthocyanins, was investigated in two thermal processing procedures of blueberryfilled pastries. The results showed that stir-frying largely decreased the contents of flavonoids, especially anthocyanins, in blueberry filling, and baking caused less variation in flavonoid profiles. However, the changes in other phenolic compounds, which may be related to the degradation of flavonoids, and the components aside from polyphenols in the fillings remain largely undiscovered. The chemical changes and the underlying mechanisms during food production should be further studied. Food matrix is such a complex mixture that phenolic compounds, with their chemical reactivity, may undergo reactions with various compounds other than their own degradation. Therefore, a holistic view on the variation of phytochemicals in blueberries during processing is important to provide insights into the changes in bioactive compounds, and encourage and give hints for scientists to further evaluate the underlying chemical reaction mechanisms of key varying compounds. Such information on the effect of thermal processing on the phytochemical profiles in real food systems, such as blueberry pastry as in this case, remains largely limited.

The current study aims to provide useful information on the chemical changes in blueberry-containing products during thermal processing by using a widely targeted metabolomic approach. The results will present clues on the degradation of thermal-sensitive components and the interaction between bioactive compounds of the products during processing. The work also provides further insights into the utilization of widely targeted metabolomic techniques in the screening of key chemical changes and the revelation of underlying chemical mechanisms.

\section{MATERIALS AND METHODS}

\section{Materials and Chemicals}

Fresh blueberries (Vaccinium spp.) were imported from Chile, and all the other ingredients of blueberry-filled pastries were purchased from the local market. Methanol, acetonitrile, and acetic acid were of HPLC grade and purchased from Merck (Darmstadt, Hesse, Germany). Double deionized ultrapure water was prepared using an ultrapure-water purification system (Millipore, Bedford, MA, USA). Chemical standards were purchased from Sigma-Aldrich (St. Louis, MO, USA) and BioBioPha (Kunming, Yunnan, China).

\section{Sample Preparation for Metabolomics Analysis}

Samples from the same batch of blueberry pastry filling samples prepared according to our previous study (8) were subjected to metabolomic analysis for the understanding of phytochemical changes under different processing procedures, including the raw filling, stir-fried, and baked samples $\left(170^{\circ} \mathrm{C}, 23 \mathrm{~min}\right)$. All samples were lyophilized and crushed using a mixer mill (MM 400, Retsch, Haan, Germany) with a zirconia bead for $90 \mathrm{~s}$ at $30 \mathrm{~Hz}$. Exactly $100 \mathrm{mg}$ sample was then weighed accurately, extracted with $1 \mathrm{~mL}$ of $70 \%$ aqueous methanol overnight at $4^{\circ} \mathrm{C}$ by vigorously vortexing for three times. After centrifugation at $10,000 \times \mathrm{g}$ for $10 \mathrm{~min}$, the extracts were filtered $(0.22 \mu \mathrm{m})$ into a vial for UHPLC-MS/MS analysis. The quality control (QC) samples were prepared in triplicate by evenly mixing all the sample extracts tested and injected every six sample injections to monitor the measure repeatability.

\section{UHPLC-MS/MS Analysis}

Exactly $2 \mu \mathrm{L}$ of sample were injected and analyzed using a Shim-pack UFLC SHIMADZU CBM30A system (Shimadzu Corporation, Kyoto, Japan) interfaced to an Applied Biosystems 6500 QTRAP mass spectrometer (AB Sciex, Foster, CA, USA). The sample was separated on a Waters ACQUITY UPLC HSS T3 C18 column $(100 \mathrm{~mm} \times 2.1 \mathrm{~mm}$, particle size $1.8 \mu \mathrm{m})$ (Waters Corporation, Milford, MA, USA) by using water $(0.04 \%$ acetic acid) as solvent $\mathrm{A}$ and acetonitrile ( $0.04 \%$ acetic acid) as solvent $\mathrm{B}$. The eluting gradient programme was as follows: $0.0-11.0 \mathrm{~min}$, $5 \%-95 \% \mathrm{~B} ; 11.0-12.0 \mathrm{~min}, 95 \% \mathrm{~B} ; 12.0-12.1 \mathrm{~min}, 95 \%-5 \%$ $\mathrm{B}$; and $12.1-15.0 \mathrm{~min}, 5 \% \mathrm{~B}$. The flow rate was $0.4 \mathrm{~mL} / \mathrm{min}$. The temperature of column oven was kept at $40^{\circ} \mathrm{C}$ during the 
analysis. The effluent was connected to an ESI-triple quadrupolelinear ion trap (QQQ-LIT) mass spectrometer equipped with an ESI Turbo Ion-Spray interface operating in both positive and negative ion modes and controlled by Analyst 1.6.3 software. The ESI source operation parameters were as follows: source temperature $500^{\circ} \mathrm{C}$; ion spray voltage (IS), $5500 \mathrm{~V}$; and ion source gas I (GSI)/gas II (GSII)/curtain gas (CUR), 55/60/25 psi. Instrument tuning and mass calibration were performed with 10 and $100 \mu \mathrm{mol} / \mathrm{L}$ polypropylene glycol solutions in QQQ and LIT modes, respectively. QQQ scans were acquired as multiple reaction monitoring (MRM) experiments with collision gas set to 5 psi. Declustering potential (DP) and collision energy (CE) for individual MRM transitions were obtained with further DP and CE optimisation. A specific set of MRM transitions was monitored for each period according to the chemicals eluted within this period.

\section{Qualification and Quantification of Chemicals}

For qualification of the chemicals, the primary and secondary mass spectrometry data were analyzed using MVDB V2.0 Database (Metware Biotechnology Co., Ltd., Wuhan, China) and the public database of metabolite information. The mass error comparing with the database was limited within $\pm 0.4 \mathrm{Da}$, and the error for retention time was allowed within $\pm 0.1 \mathrm{~min}$. The interference from isotope signals, duplicate signals of $\mathrm{K}^{+}$, $\mathrm{Na}^{+}$, and $\mathrm{NH}_{4}^{+}$ions, and duplicate signals of fragment ions derived from other larger molecules were excluded. Structural analysis of chemicals was conducted in reference to the existing mass spectrometry databases, such as MassBank (http://www. massbank.jp), KNAPSAcK (http://kanaya.naist.jp/KNApSAcK), HMDB (http://www.hmdb.ca), and METLIN (http://metlin. scripps.edu/index.php). The chemicals were quantified in the MRM mode using QQQ mass spectrometry. After chemical data from different samples were obtained, the peak areas of the mass spectra of all substances were integrated, and the mass spectrum peaks of the same chemical in different samples were subjected to integration correction.

\section{Statistical Analysis}

All samples were analyzed in triplicate. To investigate the effect of different thermal processing procedures on the composition of chemicals, hierarchical cluster analysis (HCA), principal component analysis (PCA), partial least squares-discriminant analysis (PLS-DA), and univariate analysis (UVA) on different samples were conducted with the MetaboAnalyst 4.0 platform. The thresholds for screening differential chemicals were set as follows: fold change $\geq 2$ or $\leq 0.5$ and VIP $\geq 1$.

\section{RESULTS AND DISCUSSION}

Our previous study (8) showed that the flavonoid content varied considerably during the thermal processing of blueberryfilled pastries, and the procedures of stir-frying and baking contributed to the different levels of the loss of anthocyanins and flavonol glycosides. However, the degradation products of these flavonoids and the variation of the other phytochemicals were left undetected. Pastry filling is a complex food mixture that contains many components other than flavonoids, and various reactions may occur in addition to the simple degradation of flavonoids. A holistic view on the phytochemical changes during the production of blueberry-filled pastries will help us evaluate the nutritional changes during thermal processing. The metabolomic approach has been largely applied to investigate the metabolite changes during plant development, such as fruit ripening and seed germination (13-15).

This work applied metabolomic analysis to investigate the phytochemical changes of blueberry-filled pastry products during different thermal processing stages. All the samples were lyophilized and analyzed on the basis of dry weight mass to eliminate the water deviation between the samples in different thermal processing procedures.

\section{Full List of Chemical Components in Raw and Thermal-Treated Samples}

A total ion current chromatogram of QC sample (an even mixture of all the samples involved in this study) and a multi-peak detection plot of chemicals in the MRM mode of the same sample are illustrated in Supplementary Figure 1. The chemicals in the samples were qualified and quantified by mass spectrometry by using the MVDB V2.0 database. The appearance and abundance of different substances in various samples were evaluated by MRM analysis. As shown in Supplementary Figures 1C,D, each peak displaying a distinct color represents one compound in the sample analyzed.

A total of 630 compounds were identified in the current study in the blueberry fillings of pastries collected at different processed stages, including 148 flavonoids, 21 carbohydrates, 69 organic acids, 24 quinates and their derivatives, 19 benzoic acid derivatives, 17 coumarins, 29 hydroxycinnamoyl derivatives, 5 proanthocyanidins, 2 flavonolignans, 28 amino acids and 56 of their derivatives, 18 fatty acids, 16 glycerolipids, 35 glycerophospholipids, 4 nicotinic acid derivatives, 57 nucleotides and their derivatives, 16 phenolamides, 7 indole derivatives, 3 pyridine derivatives, 4 cholines, 7 alcohols and polyols, 7 alkaloids, 7 tryptamine derivatives, 3 terpenoids, and 28 other chemicals. Amongst them, the largest group was flavonoid, which consisted of 13 anthocyanins, 10 catechin derivatives, 17 flavanones, 37 flavones, 26 flavone C-glycosides, 38 flavonols, and 7 isoflavones. The name and molecular formula of compounds, their precursor and product ions for qualification and quantification with MRM, and the peak integration values of the compounds are all listed in Supplementary Table 1. To the best of our knowledge, this study was the first to utilize the targeted UHPLC-MS/MS-based metabolomic approach in analyzing the chemical variations in fruit fillings of bakery products under different thermal treatments. Such a large amount of the compositional information provides a comprehensive understanding of the chemical changes in berries commonly enriched with bioactive polyphenols when subjected to thermal processing. 


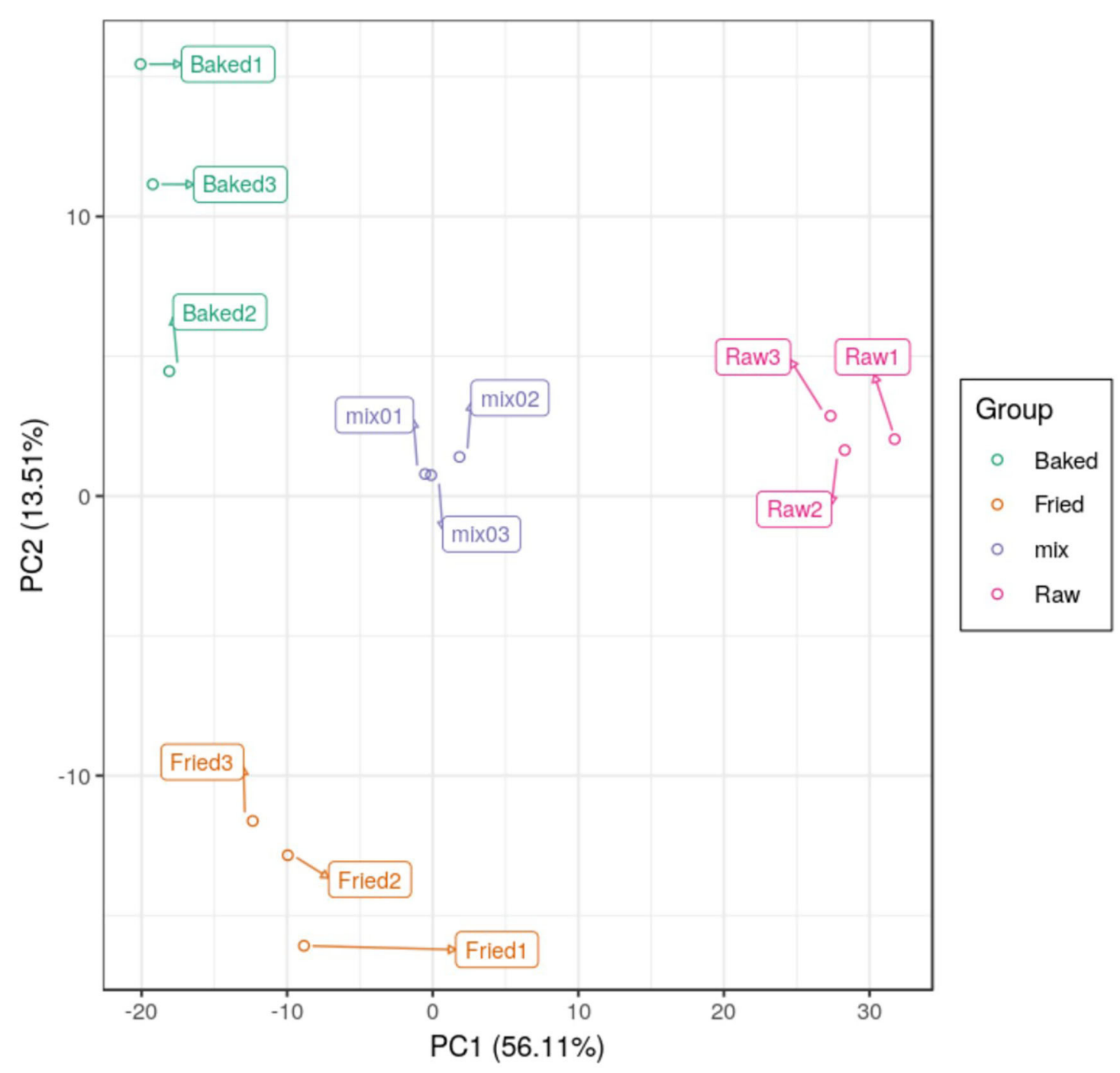

FIGURE 1 | PCA scores scatter plot showing differences in composition between blueberry filling samples obtained at different thermal processing stages. mix, quality control sample; raw, fried, and baked refer to raw, fried, and baked fillings, respectively.

\section{Difference in the Composition Amongst Raw, Stir-Fried, and Baked Blueberry Fillings}

PCA and HCA were applied to analyze the data obtained for a full view of the differences between the blueberry filling samples obtained at different processing stages. The PCA score scatter plot (Figure 1) showed that samples obtained at different processing stages could be well-distinguished. The QC samples, as a mixture of all the three samples investigated, were located in the middle of the plot. The PC1 explained $56.11 \%$ of the variances of chemical data of all the samples investigated and predominantly distinguished the raw filling samples from the thermally treated samples, such as stir-fried and baked samples. PC2 explained only $13.51 \%$ of the variances of chemical data and distinguished mainly the stir-fried filling samples from the baked filling samples. This finding indicated that stir-frying and baking dramatically changed the composition of blueberry fillings. This finding was further confirmed by the heatmap of the hierarchical clustering analysis of differential chemicals amongst samples of raw, fried, and baked fillings (Figure 2). A total of 288 differential chemicals were screened by UVA. The HCA of the 288 differential chemicals in the three samples showed clear grouping patterns. The color sequence from red to green indicates a decrease in chemical contents. The abscissa of the thermogram indicates the different sample groups, and the ordinate indicates the differential chemicals identified between groups. According to the Venn diagram shown in Figure 3, 197 and 238 chemicals significantly differed (fold change $\leq 0.5$ or $\geq 2$, and VIP $\geq 1$ ) between raw and fried fillings and between raw and baked fillings, respectively. However, the number of differential chemicals in fried filling vs. baked filling was only 75 , which confirmed our 


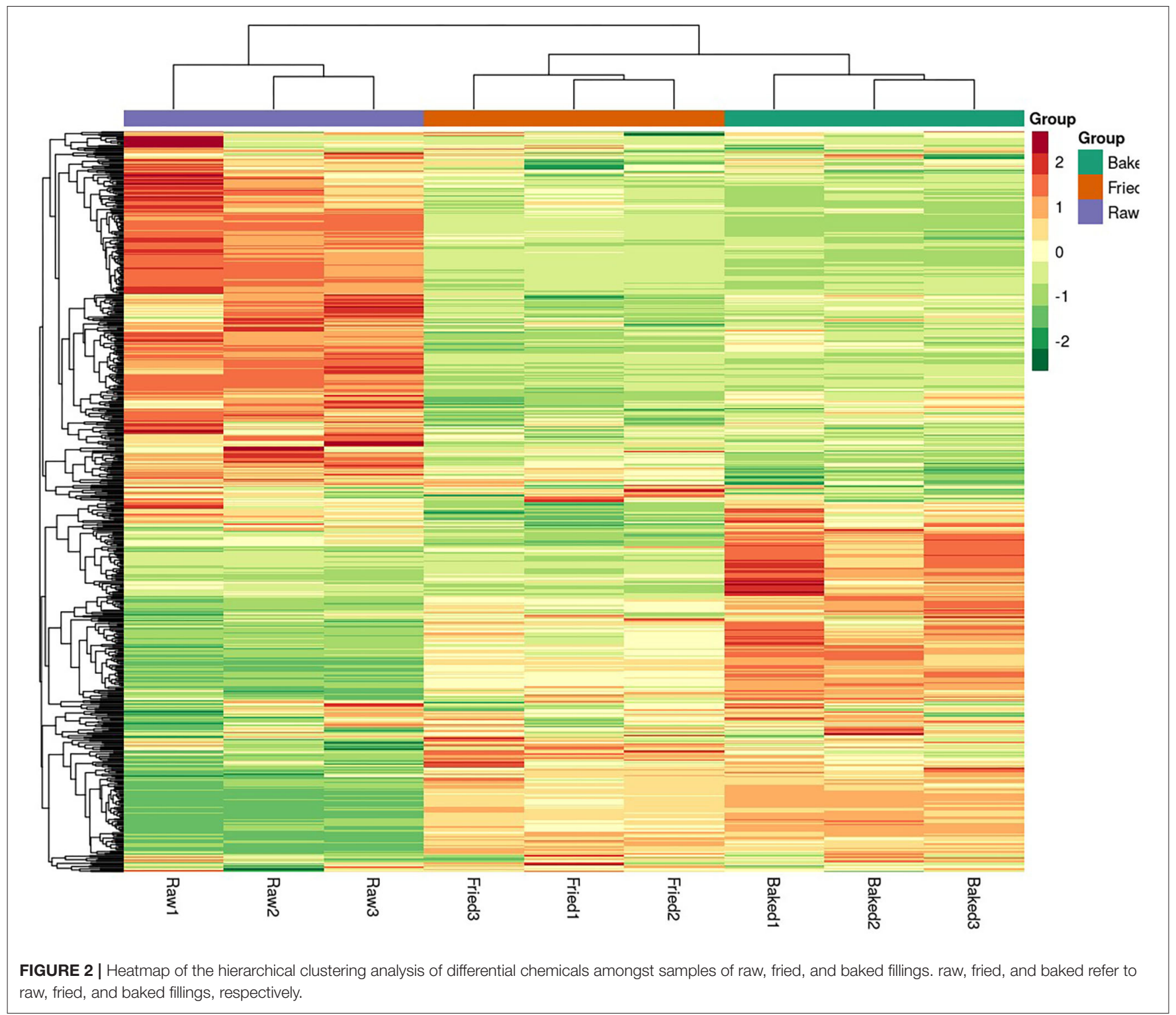

previous finding that stir-frying was the major step contributing to the most variation in chemicals during blueberry pastry preparation (8). Consistently, the heatmap of HCA showed larger differences in abundance between raw and fried fillings than those between baked and fried fillings (Figure 2).

Amongst 288 differential compounds, 117 belonged to the group of phenolic compounds (Table 1), including 18 flavones (out of 37 flavones detected), 13 anthocyanins (13), 21 flavonols (38), 12 flavone C-glycosides (26), 8 flavanones (17), 14 benzoic acid derivatives (19), 14 hydroxycinnamoyl derivatives (29), 4 catechin derivatives (10), 5 coumarins (17), 6 quinates and their derivatives (24), 1 isoflavone (7), and 1 proanthocyanidin (5). The other compounds (Table 2) included 34 nucleotides and their derivatives (57), 29 glycerophospholipids (35), 21 organic acids (69), 5 amino acids (28), 22 amino acid derivatives (56), 13 glycerolipids (16), 8 carbohydrates (21), 8 phenolamides (16), 6 fatty acids (18), 3 tryptamine derivatives (7), 3 indole derivatives (7), 2 alkaloids (7), 2 terpenoids (3), 2 nicotinic acid derivatives (4), 2 alcohols and polyols (7), 1 pyridine derivative (3), and 1 choline (4). However, the flavonolignans detected in the fillings did not vary during the processing of pastries.

\section{Variation in the Phenolic Composition of Blueberry Fillings During Thermal Processing}

All the anthocyanins showed decreasing trends during the preparation of blueberry-filled pastries, and the most variation occurred at the stir-frying stage. This result was in accordance with our previous finding (8) in which anthocyanins were the most sensitive toward stir-frying and less sensitive to baking, which should be most likely attributed to the higher temperature 


\section{Venn diagram}

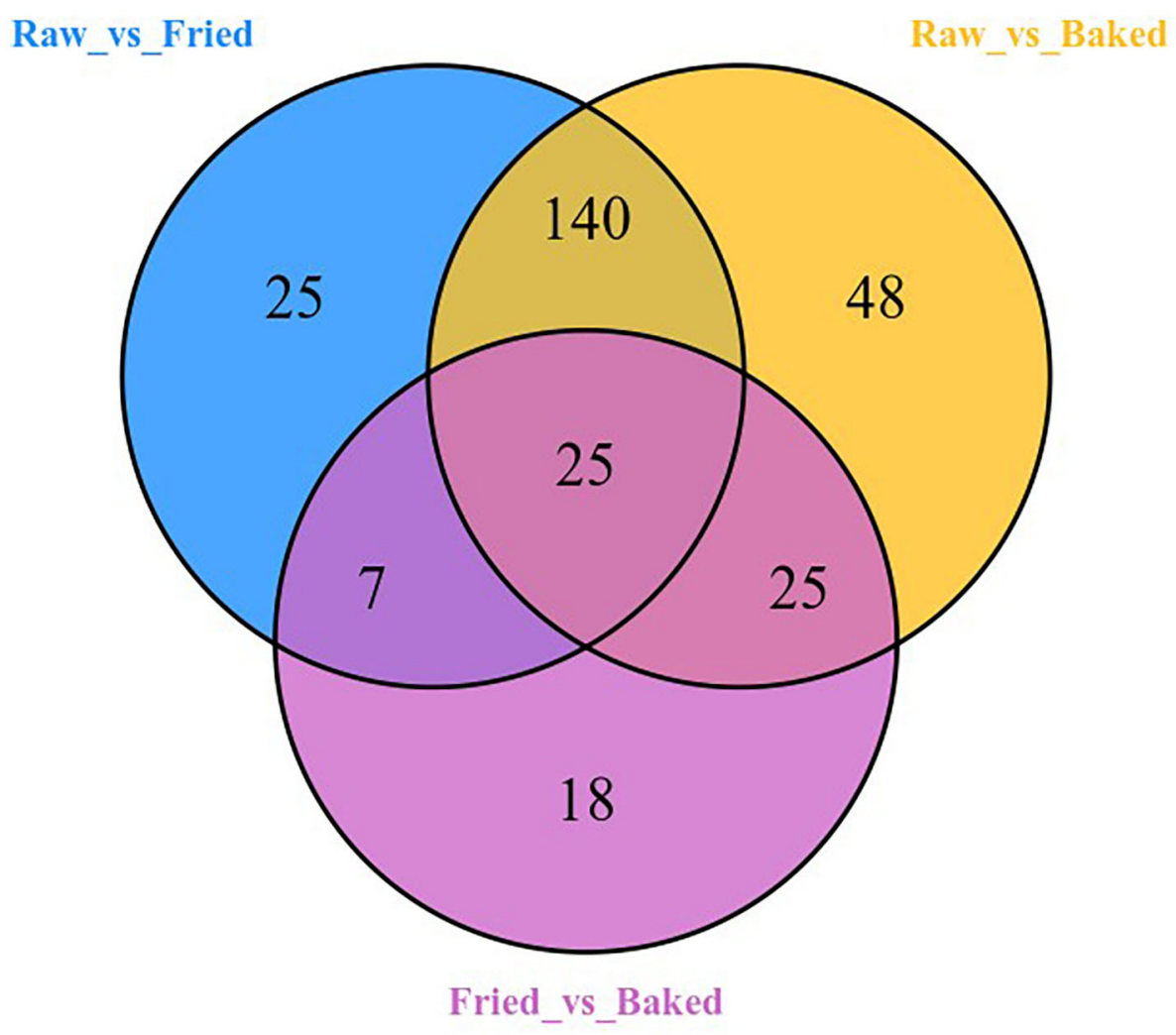

FIGURE 3 | Venn diagram of the differential compounds of blueberry fillings at different processing stages. raw, fried, and baked refer to raw, fried, and baked fillings, respectively.

and longer period of heating occurred during stir-firing than baking. Moreover, the low concentration of oxygen in the sealed fillings might decrease the reduction in flavonoids under baking conditions (16), while abundant air perfused into the fillings and accelerated the degradation of flavonoids during stir-frying.

Except for cyanidin 3-O-malonylhexoside, the contents of all the anthocyanins decreased by $52-100 \%$ during stir-frying in the current study. However, during baking, cyanidin 3-Omalonylhexoside along with four other anthocyanins, namely cyanidin 3-O-galactoside, malvidin 3-O-galactoside, delphinidin 3-O-galactoside, and petunidin 3-O-galactoside, decreased by $52-67 \%$. Cyanidin 3,5-O-diglucoside was neither detected in the fried filling nor in the baked filling. Therefore, it was totally degraded after thermal treatment. Cyanidin 3,5-O-diglucoside was identified by Hou et al. (17) in the anthocyanin-rich extract from black rice and was found to be comparable to the other anthocyanins detected in terms of thermal sensitivity because of the low values of activation energies $\left(E_{a}\right)$. CevallosCasals and Cisneros-Zevallos (18) suggested that sugars and their degradation products might accelerate the degradation of anthocyanins because of the association of the degradation rate of anthocyanin with the degradation rate of sugars to furfuraltype compounds derived from the Maillard reaction. 2-Furoic acid, which is an oxidation product of furfural that originated from the decomposition of sugars, and ascorbic acid $(19,20)$ was found to be produced in the fillings after thermal processing (Table 1). Moreover, Louarme and Billaud (21) demonstrated that the production of 2 -furoic acid depended on the oxidative degradation reactions of ascorbic acid rather than the thermal degradations, which might explain the remarkable occurrence of the compound during stir-frying (high oxygen exposure and long heating period) but mild changes under baking (low oxygen exposure in the sealed fillings and short period of heating).

Some flavonol aglycones detected in the current study increased considerably after thermal treatment by stir-frying, accompanying the decreases in the contents of their glycosides (Table 1). For example, the abundance of quercetin increased 
TABLE 1 | List of differential chemicals belonging to phenolic compounds and their variations under different thermal processing procedures.

\begin{tabular}{|c|c|c|c|c|c|c|c|c|c|c|c|c|c|}
\hline \multirow[t]{2}{*}{ Chemical } & \multirow[t]{2}{*}{ Class } & \multicolumn{4}{|c|}{ Fried filling vs. raw filling } & \multicolumn{4}{|c|}{ Baked filling vs. fried filling } & \multicolumn{4}{|c|}{ Baked filling vs. raw filling } \\
\hline & & $\begin{array}{l}\text { Variation } \\
\text { percentage } \\
(\%)\end{array}$ & VIP & $\begin{array}{l}\text { Fold } \\
\text { change }\end{array}$ & $\begin{array}{l}\text { Up/down } \\
\text { regulated }\end{array}$ & $\begin{array}{l}\text { Variation } \\
\text { percentage } \\
(\%)\end{array}$ & VIP & $\begin{array}{l}\text { Fold } \\
\text { change }\end{array}$ & $\begin{array}{l}\text { Up/down } \\
\text { regulated }\end{array}$ & $\begin{array}{l}\text { Variation } \\
\text { percentage } \\
(\%)\end{array}$ & VIP & $\begin{array}{l}\text { Fold } \\
\text { change }\end{array}$ & $\begin{array}{l}\text { Up/down } \\
\text { regulated }\end{array}$ \\
\hline Peonidin O-hexoside & Anthocyanins & $-69.84 \%$ & 1.20 & 0.30 & Down & & & & - & $-83.68 \%$ & 1.24 & 0.16 & Down \\
\hline Cyanidin 3-O-malonylhexoside & Anthocyanins & & & & - & $-66.66 \%$ & 1.11 & 0.00 & Down & $-100.00 \%$ & 1.24 & 0.00 & Down \\
\hline Rosinidin O-hexoside & Anthocyanins & $-73.88 \%$ & 1.15 & 0.26 & Down & & & & - & $-80.74 \%$ & 1.22 & 0.19 & Down \\
\hline Cyanidin 3-O-galactoside & Anthocyanins & $-76.09 \%$ & 1.20 & 0.24 & Down & $-54.24 \%$ & 1.42 & 0.46 & Down & $-89.01 \%$ & 1.22 & 0.11 & Down \\
\hline Peonidin & Anthocyanins & $-52.49 \%$ & 1.13 & 0.47 & Down & & & & - & $-57.59 \%$ & 1.22 & 0.42 & Down \\
\hline Cyanidin O-syringic acid & Anthocyanins & $-80.69 \%$ & 1.20 & 0.19 & Down & & & & - & $-88.68 \%$ & 1.23 & 0.11 & Down \\
\hline Delphinidin & Anthocyanins & $-68.14 \%$ & 1.18 & 0.32 & Down & & & & - & & & & - \\
\hline Malvidin 3-O-galactoside & Anthocyanins & $-69.24 \%$ & 1.20 & 0.31 & Down & $-51.86 \%$ & 1.42 & 0.49 & Down & $-85.07 \%$ & 1.22 & 0.15 & Down \\
\hline Malvidin 3-O-glucoside & Anthocyanins & $-71.62 \%$ & 1.21 & 0.28 & Down & & & & - & $-84.92 \%$ & 1.22 & 0.15 & Down \\
\hline Delphinidin 3-O-galactoside & Anthocyanins & $-81.20 \%$ & 1.20 & 0.19 & Down & $-53.80 \%$ & 1.40 & 0.46 & Down & $-91.23 \%$ & 1.22 & 0.09 & Down \\
\hline Cyanidin 3,5-O-diglucoside & Anthocyanins & $-100.00 \%$ & 1.21 & 0.00 & Down & & & & - & $-100.00 \%$ & 1.24 & 0.00 & Down \\
\hline Petunidin 3-O-galactoside & Anthocyanins & $-70.94 \%$ & 1.20 & 0.29 & Down & $-55.12 \%$ & 1.43 & 0.45 & Down & $-86.81 \%$ & 1.22 & 0.13 & Down \\
\hline Pelargonidin 3-O-beta-D-glucoside & Anthocyanins & $-71.03 \%$ & 1.20 & 0.29 & Down & & & & - & $-81.46 \%$ & 1.24 & 0.18 & Down \\
\hline p-Aminobenzoate & $\begin{array}{l}\text { Benzoic acid } \\
\text { derivatives }\end{array}$ & & & & - & & & & - & $110.35 \%$ & 1.15 & 2.10 & Up \\
\hline $\begin{array}{l}\text { 8-Methyl-2-oxo-4-phenyl-2H- } \\
\text { chromen-7-yl } \\
\text { 4-(hexyloxy)benzoate }\end{array}$ & $\begin{array}{l}\text { Benzoic acid } \\
\text { derivatives }\end{array}$ & $-66.26 \%$ & 1.16 & 0.34 & Down & & & & - & $-68.53 \%$ & 1.20 & 0.30 & Down \\
\hline Methyl gallate & $\begin{array}{l}\text { Benzoic acid } \\
\text { derivatives }\end{array}$ & $240.94 \%$ & 1.18 & 3.39 & Up & & & & - & $244.30 \%$ & 1.22 & 3.38 & Up \\
\hline Vanillin & $\begin{array}{l}\text { Benzoic acid } \\
\text { derivatives }\end{array}$ & $154.28 \%$ & 1.13 & 2.43 & Up & & & & - & $189.27 \%$ & 1.15 & 2.66 & Up \\
\hline Gallic acid & $\begin{array}{l}\text { Benzoic acid } \\
\text { derivatives }\end{array}$ & $504.62 \%$ & 1.20 & 6.05 & Up & & & & - & $614.96 \%$ & 1.24 & 7.11 & Up \\
\hline 2,5-dihydroxybenzoic acid & $\begin{array}{l}\text { Benzoic acid } \\
\text { derivatives }\end{array}$ & $383.65 \%$ & 1.19 & 4.78 & Up & & & & - & $528.36 \%$ & 1.23 & 6.08 & Up \\
\hline
\end{tabular}


TABLE 1 | Continued

\begin{tabular}{|c|c|c|c|c|c|c|c|c|c|c|c|c|c|}
\hline \multirow[t]{2}{*}{ Chemical } & \multirow[t]{2}{*}{ Class } & \multicolumn{4}{|c|}{ Fried filling vs. raw filling } & \multicolumn{4}{|c|}{ Baked filling vs. fried filling } & \multicolumn{4}{|c|}{ Baked filling vs. raw filling } \\
\hline & & $\begin{array}{c}\text { Variation } \\
\text { percentage } \\
(\%)\end{array}$ & VIP & $\begin{array}{c}\text { Fold } \\
\text { change }\end{array}$ & $\begin{array}{l}\text { Up/down } \\
\text { regulated }\end{array}$ & $\begin{array}{c}\text { Variation } \\
\text { percentage } \\
(\%)\end{array}$ & VIP & $\begin{array}{c}\text { Fold } \\
\text { change }\end{array}$ & $\begin{array}{l}\text { Up/down } \\
\text { regulated }\end{array}$ & $\begin{array}{c}\text { Variation } \\
\text { percentage } \\
(\%)\end{array}$ & VIP & $\begin{array}{c}\text { Fold } \\
\text { change }\end{array}$ & $\begin{array}{l}\text { Up/down } \\
\text { regulated }\end{array}$ \\
\hline 4-Hydroxybenzaldehyde & $\begin{array}{l}\text { Benzoic acid } \\
\text { derivatives }\end{array}$ & $157.09 \%$ & 1.19 & 2.57 & Up & & & & - & $274.27 \%$ & 1.23 & 3.70 & Up \\
\hline 2,3-Dihydroxybenzoic acid & $\begin{array}{l}\text { Benzoic acid } \\
\text { derivatives }\end{array}$ & $438.59 \%$ & 1.20 & 5.39 & Up & & & & & $584.63 \%$ & 1.24 & 6.77 & Up \\
\hline 4-Hydroxybenzoic acid & $\begin{array}{l}\text { Benzoic acid } \\
\text { derivatives }\end{array}$ & & & & - & & & & & $123.11 \%$ & 1.20 & 2.18 & Up \\
\hline 2-(Formylamino)benzoic acid & $\begin{array}{l}\text { Benzoic acid } \\
\text { derivatives }\end{array}$ & $143.61 \%$ & 1.16 & 2.39 & Up & & & & & $207.25 \%$ & 1.20 & 2.95 & Up \\
\hline Anthranilic acid & $\begin{array}{l}\text { Benzoic acid } \\
\text { derivatives }\end{array}$ & $167.46 \%$ & 1.18 & 2.65 & Up & & & & - & $166.59 \%$ & 1.20 & 2.63 & Up \\
\hline Syringaldehyde & $\begin{array}{l}\text { Benzoic acid } \\
\text { derivatives }\end{array}$ & $202.84 \%$ & 1.20 & 3.03 & Up & & & & - & $245.88 \%$ & 1.24 & 3.46 & Up \\
\hline Vanillic acid & $\begin{array}{l}\text { Benzoic acid } \\
\text { derivatives }\end{array}$ & $172.84 \%$ & 1.17 & 2.69 & Up & & & & - & $225.36 \%$ & 1.21 & 3.13 & Up \\
\hline Syringic acid & $\begin{array}{l}\text { Benzoic acid } \\
\text { derivatives }\end{array}$ & $854.07 \%$ & 1.20 & 9.52 & Up & & & & - & $1072.42 \%$ & 1.24 & 11.58 & Up \\
\hline Gallocatechin-gallocatechin & $\begin{array}{l}\text { Catechin } \\
\text { derivatives }\end{array}$ & & & & - & $-100.00 \%$ & 1.62 & 0.00 & Down & $-100.00 \%$ & 1.24 & 0.00 & Down \\
\hline Epigallocatechin & $\begin{array}{l}\text { Catechin } \\
\text { derivatives }\end{array}$ & $590.56 \%$ & 1.20 & 6.85 & Up & & & & - & $511.38 \%$ & 1.24 & 6.09 & Up \\
\hline Protocatechuic acid & $\begin{array}{l}\text { Catechin } \\
\text { derivatives }\end{array}$ & $429.20 \%$ & 1.20 & 5.29 & Up & & & & - & $575.14 \%$ & 1.24 & 6.70 & Up \\
\hline Protocatechuic aldehyde & $\begin{array}{l}\text { Catechin } \\
\text { derivatives }\end{array}$ & $378.86 \%$ & 1.20 & 4.72 & Up & & & & - & $439.77 \%$ & 1.23 & 5.25 & Up \\
\hline O-Feruloyl 4-hydroxylcoumarin & Coumarins & $-58.15 \%$ & 1.18 & 0.41 & Down & & & & - & $-60.44 \%$ & 1.14 & 0.39 & Down \\
\hline $\begin{array}{l}\text { 6,7-dihydroxycoumarin 7-O-quinic } \\
\text { acid }\end{array}$ & Coumarins & $-48.02 \%$ & 1.08 & 0.50 & Down & & & & - & & & & - \\
\hline 7-Hydroxy-5-methoxycoumarin & Coumarins & & & & - & & & & - & $205.85 \%$ & 1.22 & 2.98 & Up \\
\hline 6-Methoxy-7,8-dihydroxycoumarin & Coumarins & $1319.84 \%$ & 1.13 & 10.17 & Up & $126.94 \%$ & 1.59 & 2.21 & Up & $3402.35 \%$ & 1.20 & 22.46 & Up \\
\hline Scoparone & Coumarins & & & & - & $-66.62 \%$ & 1.18 & 0.00 & Down & $-99.96 \%$ & 1.24 & 0.00 & Down \\
\hline Naringenin O-malonylhexoside & Flavanone & $-68.07 \%$ & 1.14 & 0.32 & Down & & & & - & $-72.48 \%$ & 1.18 & 0.27 & Down \\
\hline 4'-Hydroxy-5,7-dimethoxyflavanone & Flavanone & $114.58 \%$ & 1.12 & 2.12 & Up & & & & - & & & & - \\
\hline Naringenin & Flavanone & $236.74 \%$ & 1.17 & 3.29 & Up & & & & - & $369.93 \%$ & 1.22 & 4.57 & Up \\
\hline
\end{tabular}


TABLE 1 | Continued

\begin{tabular}{|c|c|c|c|c|c|c|c|c|c|c|c|c|c|}
\hline \multirow[t]{2}{*}{ Chemical } & \multirow[t]{2}{*}{ Class } & \multicolumn{4}{|c|}{ Fried filling vs. raw filling } & \multicolumn{4}{|c|}{ Baked filling vs. fried filling } & \multicolumn{4}{|c|}{ Baked filling vs. raw filling } \\
\hline & & $\begin{array}{c}\text { Variation } \\
\text { percentage } \\
(\%)\end{array}$ & VIP & $\begin{array}{l}\text { Fold } \\
\text { change }\end{array}$ & $\begin{array}{l}\text { Up/down } \\
\text { regulated }\end{array}$ & $\begin{array}{c}\text { Variation } \\
\text { percentage } \\
(\%)\end{array}$ & VIP & $\begin{array}{l}\text { Fold } \\
\text { change }\end{array}$ & $\begin{array}{l}\text { Up/down } \\
\text { regulated }\end{array}$ & $\begin{array}{c}\text { Variation } \\
\text { percentage } \\
(\%)\end{array}$ & VIP & $\begin{array}{l}\text { Fold } \\
\text { change }\end{array}$ & $\begin{array}{l}\text { Up/down } \\
\text { regulated }\end{array}$ \\
\hline Phloretin & Flavanone & & & & - & & & & - & $113.43 \%$ & 1.20 & 2.14 & Up \\
\hline Eriodictyol & Flavanone & & & & - & & & & - & $150.83 \%$ & 1.23 & 2.50 & Up \\
\hline Naringenin chalcone & Flavanone & $235.51 \%$ & 1.16 & 3.30 & Up & & & & - & $373.97 \%$ & 1.22 & 4.63 & Up \\
\hline sakuranetin & Flavanone & + & 1.02 & 28.50 & Up & & & & - & + & 1.04 & 34.41 & Up \\
\hline 3,5,7,4'-Tetrahydroxyflavan & Flavanone & & & & - & & & & - & $-62.12 \%$ & 1.17 & 0.38 & Down \\
\hline Selgin O-malonylhexoside & Flavone & $-65.72 \%$ & 1.19 & 0.34 & Down & & & & - & $-70.87 \%$ & 1.21 & 0.29 & Down \\
\hline Chrysin O-malonylhexoside & Flavone & $-68.60 \%$ & 1.17 & 0.32 & Down & & & & - & & & & - \\
\hline Spinacetin & Flavone & $-53.97 \%$ & 1.18 & 0.46 & Down & & & & - & & & & - \\
\hline Chrysoeriol 7-O-rutinoside & Flavone & $-55.04 \%$ & 1.19 & 0.45 & Down & & & & - & $-49.31 \%$ & 1.15 & 0.49 & Down \\
\hline Chrysoeriol O-hexosyl-O-hexoside & Flavone & $-54.84 \%$ & 1.17 & 0.45 & Down & & & & - & $-63.23 \%$ & 1.22 & 0.37 & Down \\
\hline Apigenin 7-O-glucoside & Flavone & & & & - & & & & - & $-59.36 \%$ & 1.01 & 0.31 & Down \\
\hline Tricin 5-O-hexosyl-O-hexoside & Flavone & + & 1.21 & 79789.26 & Up & & & & - & + & 1.24 & 156066.67 & Up \\
\hline Tricin O-rhamnoside & Flavone & $-99.96 \%$ & 1.20 & 0.00 & Down & & & & - & & & & - \\
\hline Tricin 5-O-rutinoside & Flavone & $-85.13 \%$ & 1.18 & 0.15 & Down & & & & - & $-91.00 \%$ & 1.23 & 0.09 & Down \\
\hline Tricin O-hexosyl-O-syringin alcohol & Flavone & + & 1.21 & 1511.37 & Up & & & & - & + & 1.24 & 1384.53 & Up \\
\hline Tricin & Flavone & & & & - & & & & - & $119.60 \%$ & 1.18 & 2.18 & Up \\
\hline Acacetin $O$-acetyl hexoside & Flavone & & & & - & $124.85 \%$ & 1.45 & 2.10 & Up & & & & - \\
\hline Chrysoeriol O-acetylhexoside & Flavone & $-49.75 \%$ & 1.10 & 0.47 & Down & & & & - & & & & - \\
\hline Apigenin 7-O-neohesperidoside & Flavone & & & & - & & & & - & $135.33 \%$ & 1.18 & 2.29 & Up \\
\hline Chrysoeriol & Flavone & & & & - & & & & - & $174.73 \%$ & 1.20 & 2.75 & Up \\
\hline Nobiletin & Flavone & & & & - & $-37.89 \%$ & 1.10 & 0.49 & Down & $-76.47 \%$ & 1.21 & 0.23 & Down \\
\hline Tangeretin & Flavone & $-51.21 \%$ & 1.01 & 0.50 & Down & & & & - & $-67.96 \%$ & 1.19 & 0.30 & Down \\
\hline
\end{tabular}

(Continued) 
TABLE 1 | Continued

\begin{tabular}{|c|c|c|c|c|c|c|c|c|c|c|c|c|c|}
\hline \multirow[t]{2}{*}{ Chemical } & \multirow[t]{2}{*}{ Class } & \multicolumn{4}{|c|}{ Fried filling vs. raw filling } & \multicolumn{4}{|c|}{ Baked filling vs. fried filling } & \multicolumn{4}{|c|}{ Baked filling vs. raw filling } \\
\hline & & $\begin{array}{c}\text { Variation } \\
\text { percentage } \\
(\%)\end{array}$ & VIP & $\begin{array}{l}\text { Fold } \\
\text { change }\end{array}$ & $\begin{array}{l}\text { Up/down } \\
\text { regulated }\end{array}$ & $\begin{array}{c}\text { Variation } \\
\text { percentage } \\
(\%)\end{array}$ & VIP & $\begin{array}{l}\text { Fold } \\
\text { change }\end{array}$ & $\begin{array}{l}\text { Up/down } \\
\text { regulated }\end{array}$ & $\begin{array}{c}\text { Variation } \\
\text { percentage } \\
(\%)\end{array}$ & VIP & $\begin{array}{l}\text { Fold } \\
\text { change }\end{array}$ & $\begin{array}{l}\text { Up/down } \\
\text { regulated }\end{array}$ \\
\hline Butin & Flavone & $240.50 \%$ & 1.16 & 3.31 & Up & & & & - & $369.59 \%$ & 1.21 & 4.53 & Up \\
\hline C-hexosyl-chrysoeriol O-hexoside & $\begin{array}{l}\text { Flavone } \\
\text { C-glycosides }\end{array}$ & $-63.15 \%$ & 1.20 & 0.37 & Down & & & & - & $-69.53 \%$ & 1.24 & 0.30 & Down \\
\hline $\begin{array}{l}\text { Chrysoeriol 6-C-hexoside } \\
\text { 8-C-hexoside-O-hexoside }\end{array}$ & $\begin{array}{l}\text { Flavone } \\
\text { C-glycosides }\end{array}$ & & & & - & & & & - & $-48.82 \%$ & 1.07 & 0.47 & Down \\
\hline 6-C-hexosyl-hesperetin O-hexoside & $\begin{array}{l}\text { Flavone } \\
\text { C-glycosides }\end{array}$ & $-63.02 \%$ & 1.19 & 0.36 & Down & & & & - & $-63.59 \%$ & 1.14 & 0.37 & Down \\
\hline di-C,C-hexosyl-apigenin & $\begin{array}{l}\text { Flavone } \\
\text { C-glycosides }\end{array}$ & $-57.05 \%$ & 1.19 & 0.43 & Down & & & & - & $-73.00 \%$ & 1.24 & 0.27 & Down \\
\hline $\begin{array}{l}\text { C-hexosyl-luteolin } \\
\text { O-p-coumaroylhexoside }\end{array}$ & $\begin{array}{l}\text { Flavone } \\
\text { C-glycosides }\end{array}$ & & & & - & & & & - & $-53.56 \%$ & 1.07 & 0.46 & Down \\
\hline Chrysoeriol C-hexosyl-O-rhamnoside & $\begin{array}{l}\text { Flavone } \\
\text { C-glycosides }\end{array}$ & $-75.75 \%$ & 1.20 & 0.24 & Down & & & & - & $-86.98 \%$ & 1.24 & 0.13 & Down \\
\hline 8-C-hexosyl-luteolin O-hexoside & $\begin{array}{l}\text { Flavone } \\
\text { C-glycosides }\end{array}$ & $-65.15 \%$ & 1.15 & 0.35 & Down & & & & - & $-69.36 \%$ & 1.20 & 0.30 & Down \\
\hline Luteolin 8-C-hexosyl-O-hexoside & $\begin{array}{l}\text { Flavone } \\
\text { C-glycosides }\end{array}$ & $-52.23 \%$ & 1.16 & 0.48 & Down & & & & - & $-59.09 \%$ & 1.22 & 0.41 & Down \\
\hline C-hexosyl-apigenin C-pentoside & $\begin{array}{l}\text { Flavone } \\
\text { C-glycosides }\end{array}$ & $-55.05 \%$ & 1.16 & 0.44 & Down & & & & - & & & & - \\
\hline 8-C-hexosyl chrysoeriol O-hexoside & $\begin{array}{l}\text { Flavone } \\
\text { C-glycosides }\end{array}$ & + & 1.21 & 7587.37 & Up & & & & - & + & 1.24 & 11791.56 & Up \\
\hline Hesperetin C-hexoside O-hexoside & $\begin{array}{l}\text { Flavone } \\
\text { C-glycosides }\end{array}$ & $12525.71 \%$ & 1.20 & 122.18 & Up & & & & - & $22757.50 \%$ & 1.24 & 225.37 & Up \\
\hline Luteolin C-hexoside & $\begin{array}{l}\text { Flavone } \\
\text { C-glycosides }\end{array}$ & & & & - & & & & - & $100.60 \%$ & 1.07 & 2.03 & Up \\
\hline methylQuercetin O-hexoside & Flavonol & $-65.77 \%$ & 1.12 & 0.35 & Down & & & & - & $-75.25 \%$ & 1.20 & 0.24 & Down \\
\hline Quercetin-3,4'-O-diglucoside & Flavonol & $-74.25 \%$ & 1.19 & 0.25 & Down & & & & - & $-74.92 \%$ & 1.23 & 0.25 & Down \\
\hline Isorhamnetin O-hexoside & Flavonol & $-53.44 \%$ & 1.20 & 0.47 & Down & & & & - & $-54.86 \%$ & 1.24 & 0.45 & Down \\
\hline Isorhamnetin 5-O-hexoside & Flavonol & $-53.71 \%$ & 1.19 & 0.46 & Down & & & & - & $-54.57 \%$ & 1.22 & 0.45 & Down \\
\hline $\begin{array}{l}\text { Quercetin } \\
\text { 7-O-malonylhexosyl-hexoside }\end{array}$ & Flavonol & $-50.51 \%$ & 1.14 & 0.49 & Down & & & & - & $-60.68 \%$ & 1.16 & 0.38 & Down \\
\hline Kaempferol & Flavonol & & & & - & & & & - & $260.46 \%$ & 1.23 & 3.59 & Up \\
\hline Quercetin & Flavonol & $250.40 \%$ & 1.20 & 3.50 & Up & & & & - & $457.89 \%$ & 1.24 & 5.58 & Up \\
\hline
\end{tabular}


TABLE 1 | Continued

\begin{tabular}{|c|c|c|c|c|c|c|c|c|c|c|c|c|c|}
\hline \multirow[t]{2}{*}{ Chemical } & \multirow[t]{2}{*}{ Class } & \multicolumn{4}{|c|}{ Fried filling vs. raw filling } & \multicolumn{4}{|c|}{ Baked filling vs. fried filling } & \multicolumn{4}{|c|}{ Baked filling vs. raw filling } \\
\hline & & $\begin{array}{c}\text { Variation } \\
\text { percentage } \\
(\%)\end{array}$ & VIP & $\begin{array}{l}\text { Fold } \\
\text { change }\end{array}$ & $\begin{array}{l}\text { Up/down } \\
\text { regulated }\end{array}$ & $\begin{array}{c}\text { Variation } \\
\text { percentage } \\
(\%)\end{array}$ & VIP & $\begin{array}{l}\text { Fold } \\
\text { change }\end{array}$ & $\begin{array}{l}\text { Up/down } \\
\text { regulated }\end{array}$ & $\begin{array}{c}\text { Variation } \\
\text { percentage } \\
(\%)\end{array}$ & VIP & $\begin{array}{l}\text { Fold } \\
\text { change }\end{array}$ & $\begin{array}{l}\text { Up/down } \\
\text { regulated }\end{array}$ \\
\hline Quercetin-3-arabinoside & Flavonol & $-58.34 \%$ & 1.20 & 0.42 & Down & & & & - & $-62.80 \%$ & 1.24 & 0.37 & Down \\
\hline Kaempferol 3-O-rutinoside & Flavonol & & & & - & & & & - & $-55.41 \%$ & 1.22 & 0.44 & Down \\
\hline Myricetin & Flavonol & & & & - & & & & - & $132.31 \%$ & 1.23 & 2.30 & Up \\
\hline Dihydroquercetin & Flavonol & $141.38 \%$ & 1.05 & 2.32 & Up & & & & - & $177.36 \%$ & 1.14 & 2.56 & Up \\
\hline Isorhamnetin & Flavonol & $379.09 \%$ & 1.18 & 4.83 & Up & & & & - & $809.31 \%$ & 1.24 & 9.08 & Up \\
\hline Kaempferol 3-O-robinobioside & Flavonol & & & & - & & & & - & $-52.56 \%$ & 1.23 & 0.47 & Down \\
\hline Kaempferol 3,7-dirhamnoside & Flavonol & & & & - & & & & - & $-55.36 \%$ & 1.13 & 0.43 & Down \\
\hline Kaempferol 3-O-galactoside & Flavonol & $-60.27 \%$ & 1.19 & 0.40 & Down & & & & - & $-63.19 \%$ & 1.24 & 0.37 & Down \\
\hline 7-O-methxyl quercetin & Flavonol & + & 1.07 & 40.97 & Up & & & & - & + & 1.13 & 77.36 & Up \\
\hline Syringetin & Flavonol & $865.90 \%$ & 1.19 & 9.65 & Up & & & & - & $1433.02 \%$ & 1.24 & 14.75 & Up \\
\hline Laricitrin & Flavonol & $291.91 \%$ & 1.19 & 3.93 & Up & & & & - & $524.32 \%$ & 1.23 & 6.16 & Up \\
\hline Quercetin 7-O- $\beta$-D-Glucuronide & Flavonol & $-69.41 \%$ & 1.12 & 0.31 & Down & & & & - & $-72.95 \%$ & 1.21 & 0.27 & Down \\
\hline $\begin{array}{l}\text { Kaempferol-3-O-robinoside-7-O- } \\
\text { rhamnoside }\end{array}$ & Flavonol & $-62.14 \%$ & 1.18 & 0.38 & Down & & & & - & $-62.38 \%$ & 1.16 & 0.37 & Down \\
\hline Morin & Flavonol & $255.78 \%$ & 1.18 & 3.58 & Up & & & & - & $461.44 \%$ & 1.24 & 5.59 & Up \\
\hline 6-Hydroxymethylherniarin & $\begin{array}{l}\text { Hydroxycinnamoyl } \\
\text { derivatives }\end{array}$ & & & & - & & & & - & $-50.96 \%$ & 1.23 & 0.49 & Down \\
\hline Hydroxy-methoxycinnamate & $\begin{array}{l}\text { Hydroxycinnamoyl } \\
\text { derivatives }\end{array}$ & & & & - & & & & - & $-52.88 \%$ & 1.24 & 0.47 & Down \\
\hline 4-Methoxycinnamic acid & $\begin{array}{l}\text { Hydroxycinnamoyl } \\
\text { derivatives }\end{array}$ & $-71.75 \%$ & 1.15 & 0.28 & Down & & & & - & $-71.58 \%$ & 1.19 & 0.28 & Down \\
\hline Cinnamic acid & $\begin{array}{l}\text { Hydroxycinnamoyl } \\
\text { derivatives }\end{array}$ & $132.86 \%$ & 1.11 & 2.32 & Up & & & & - & $239.69 \%$ & 1.19 & 3.28 & Up \\
\hline Ferulic acid & $\begin{array}{l}\text { Hydroxycinnamoyl } \\
\text { derivatives }\end{array}$ & $138.88 \%$ & 1.18 & 2.38 & Up & & & & - & $207.47 \%$ & 1.23 & 3.05 & Up \\
\hline Homovanillic acid & $\begin{array}{l}\text { Hydroxycinnamoyl } \\
\text { derivatives }\end{array}$ & $-49.78 \%$ & 1.13 & 0.49 & Down & & & & - & & & & - \\
\hline 3-Hydroxy-4-methoxycinnamic acid & $\begin{array}{l}\text { Hydroxycinnamoyl } \\
\text { derivatives }\end{array}$ & $148.04 \%$ & 1.17 & 2.48 & Up & & & & - & $211.79 \%$ & 1.23 & 3.08 & Up \\
\hline
\end{tabular}


TABLE 1 | Continued

\begin{tabular}{|c|c|c|c|c|c|c|c|c|c|c|c|c|c|}
\hline \multirow[t]{2}{*}{ Chemical } & \multirow[t]{2}{*}{ Class } & \multicolumn{4}{|c|}{ Fried filling vs. raw filling } & \multicolumn{4}{|c|}{ Baked filling vs. fried filling } & \multicolumn{4}{|c|}{ Baked filling vs. raw filling } \\
\hline & & $\begin{array}{c}\text { Variation } \\
\text { percentage } \\
(\%)\end{array}$ & VIP & $\begin{array}{l}\text { Fold } \\
\text { change }\end{array}$ & $\begin{array}{l}\text { Up/down } \\
\text { regulated }\end{array}$ & $\begin{array}{c}\text { Variation } \\
\text { percentage } \\
(\%)\end{array}$ & VIP & $\begin{array}{l}\text { Fold } \\
\text { change }\end{array}$ & $\begin{array}{l}\text { Up/down } \\
\text { regulated }\end{array}$ & $\begin{array}{c}\text { Variation } \\
\text { percentage } \\
(\%)\end{array}$ & VIP & $\begin{array}{l}\text { Fold } \\
\text { change }\end{array}$ & $\begin{array}{l}\text { Up/down } \\
\text { regulated }\end{array}$ \\
\hline p-Coumaric acid & $\begin{array}{l}\text { Hydroxycinnamoyl } \\
\text { derivatives }\end{array}$ & $136.18 \%$ & 1.15 & 2.34 & Up & & & & - & $201.03 \%$ & 1.21 & 2.91 & Up \\
\hline Sinapic acid & $\begin{array}{l}\text { Hydroxycinnamoyl } \\
\text { derivatives }\end{array}$ & $1014.76 \%$ & 1.20 & 11.11 & Up & & & & - & $1407.51 \%$ & 1.24 & 15.04 & Up \\
\hline Sinapyl alcohol & $\begin{array}{l}\text { Hydroxycinnamoyl } \\
\text { derivatives }\end{array}$ & $128.98 \%$ & 1.09 & 2.18 & Up & & & & - & & & & - \\
\hline p-Coumaryl alcohol & $\begin{array}{l}\text { Hydroxycinnamoyl } \\
\text { derivatives }\end{array}$ & $-99.95 \%$ & 1.20 & 0.00 & Down & + & 1.08 & 129.93 & Up & & & & - \\
\hline Sinapinaldehyde & $\begin{array}{l}\text { Hydroxycinnamoyl } \\
\text { derivatives }\end{array}$ & $186.15 \%$ & 1.11 & 2.74 & Up & & & & - & $259.93 \%$ & 1.15 & 3.47 & Up \\
\hline p-Coumaraldehyde & $\begin{array}{l}\text { Hydroxycinnamoyl } \\
\text { derivatives }\end{array}$ & $-53.49 \%$ & 1.16 & 0.45 & Down & & & & - & $-55.49 \%$ & 1.20 & 0.43 & Down \\
\hline Caffeyl alcohol & $\begin{array}{l}\text { Hydroxycinnamoyl } \\
\text { derivatives }\end{array}$ & $-54.80 \%$ & 1.10 & 0.44 & Down & & & & - & & & & - \\
\hline Glycitin & Isoflavone & $-49.66 \%$ & 1.16 & 0.50 & Down & & & & - & $-49.56 \%$ & 1.09 & 0.49 & Down \\
\hline Procyanidin A3 & Proanthocyanidins & $256.20 \%$ & 1.19 & 3.51 & Up & & & & - & $335.10 \%$ & 1.23 & 4.24 & Up \\
\hline 1-O-Feruloyl quinic acid & $\begin{array}{l}\text { Quinate and its } \\
\text { derivatives }\end{array}$ & $-56.96 \%$ & 1.19 & 0.43 & Down & & & & - & $-59.22 \%$ & 1.23 & 0.40 & Down \\
\hline O-Feruloyl quinic acid & $\begin{array}{l}\text { Quinate and its } \\
\text { derivatives }\end{array}$ & $136.07 \%$ & 1.20 & 2.36 & Up & & & & - & $153.40 \%$ & 1.23 & 2.53 & Up \\
\hline 5-O-p-Coumaroyl shikimic acid & $\begin{array}{l}\text { Quinate and its } \\
\text { derivatives }\end{array}$ & $-64.98 \%$ & 1.18 & 0.34 & Down & & & & - & $-70.22 \%$ & 1.19 & 0.30 & Down \\
\hline Eudesmoyl quinic acid & $\begin{array}{l}\text { Quinate and its } \\
\text { derivatives }\end{array}$ & $359.65 \%$ & 1.09 & 3.80 & Up & & & & - & $388.12 \%$ & 1.10 & 3.71 & Up \\
\hline $\begin{array}{l}\text { 5-O-p-coumaroyl shikimic acid } \\
\text { O-hexoside }\end{array}$ & $\begin{array}{l}\text { Quinate and its } \\
\text { derivatives }\end{array}$ & $-75.15 \%$ & 1.20 & 0.25 & Down & & & & - & $-86.58 \%$ & 1.23 & 0.13 & Down \\
\hline Chlorogenic acid methyl ester & $\begin{array}{l}\text { Quinate and its } \\
\text { derivatives }\end{array}$ & $-51.93 \%$ & 1.13 & 0.47 & Down & & & & - & $-57.72 \%$ & 1.14 & 0.39 & Down \\
\hline
\end{tabular}

+ , Newly occurred after the corresponding thermal treatment. 
TABLE 2 | List of differential chemicals other than phenolic compounds and their variations under different thermal processing procedures.

\begin{tabular}{|c|c|c|c|c|c|c|c|c|c|c|c|c|c|}
\hline \multirow[t]{2}{*}{ Chemical } & \multirow[t]{2}{*}{ Class } & \multicolumn{4}{|c|}{ Fried filling vs. raw filling } & \multicolumn{4}{|c|}{ Baked filling vs. fried filling } & \multicolumn{4}{|c|}{ Baked filling vs. raw filling } \\
\hline & & $\begin{array}{c}\text { Variation } \\
\text { percentage } \\
(\%)\end{array}$ & VIP & $\begin{array}{l}\text { Fold } \\
\text { change }\end{array}$ & $\begin{array}{l}\text { Up/Down } \\
\text { regulated }\end{array}$ & $\begin{array}{c}\text { Variation } \\
\text { percentage } \\
(\%)\end{array}$ & VIP & $\begin{array}{l}\text { Fold } \\
\text { change }\end{array}$ & $\begin{array}{l}\text { Up/Down } \\
\text { regulated }\end{array}$ & $\begin{array}{c}\text { Variation } \\
\text { percentage } \\
(\%)\end{array}$ & VIP & $\begin{array}{l}\text { Fold } \\
\text { change }\end{array}$ & $\begin{array}{l}\text { Up/Down } \\
\text { regulated }\end{array}$ \\
\hline D(-)-Threose & Carbohydrates & $-88.98 \%$ & 1.20 & 0.11 & Down & & & & - & $-86.79 \%$ & 1.23 & 0.13 & Down \\
\hline Ribulose-5-phosphate & Carbohydrates & $122.78 \%$ & 1.02 & 2.01 & Up & & & & - & & & & - \\
\hline Glucosamine & Carbohydrates & & & & - & & & & - & + & 1.01 & 55.75 & Up \\
\hline D(+)-Melezitose O-rhamnoside & Carbohydrates & $-48.78 \%$ & 1.15 & 0.50 & Down & $-58.26 \%$ & 1.58 & 0.41 & Down & $-79.40 \%$ & 1.22 & 0.21 & Down \\
\hline Trehalose 6-phosphate & Carbohydrates & & & & - & & & & - & $235.19 \%$ & 1.16 & 3.37 & Up \\
\hline $\mathrm{D}(+)$-Melezitose & Carbohydrates & & & & - & & & & - & $236.92 \%$ & 1.03 & 2.56 & Up \\
\hline D-(+)-Glucono-1,5-lactone & Carbohydrates & $122.72 \%$ & 1.20 & 2.22 & Up & & & & - & $149.99 \%$ & 1.22 & 2.50 & Up \\
\hline L-Fucose & Carbohydrates & $543.66 \%$ & 1.19 & 6.45 & Up & & & & - & $392.67 \%$ & 1.20 & 4.91 & Up \\
\hline L-Histidine & Amino acids & & & & - & & & & - & $-59.43 \%$ & 1.20 & 0.40 & Down \\
\hline L-(+)-Arginine & Amino acids & & & & - & & & & - & $-59.68 \%$ & 1.23 & 0.40 & Down \\
\hline L-Methionine & Amino acids & $-65.76 \%$ & 1.19 & 0.34 & Down & & & & - & $-64.68 \%$ & 1.24 & 0.35 & Down \\
\hline L-Glutamine & Amino acids & $-64.62 \%$ & 1.20 & 0.35 & Down & $-56.97 \%$ & 1.58 & 0.42 & Down & $-85.10 \%$ & 1.24 & 0.15 & Down \\
\hline L(+)-Ornithine & Amino acids & $-52.14 \%$ & 1.17 & 0.48 & Down & & & & - & & & & - \\
\hline Lysine butyrate & Amino acid derivatives & & & & - & + & 1.34 & 60.90 & Up & + & 1.13 & 213.85 & Up \\
\hline N-Acetylmethionine & Amino acid derivatives & $-74.42 \%$ & 1.20 & 0.26 & Down & & & & - & $-78.16 \%$ & 1.23 & 0.22 & Down \\
\hline Acetyl tryptophan & Amino acid derivatives & & & & - & & & & - & $158.90 \%$ & 1.03 & 2.53 & Up \\
\hline L-Glutamine O-hexside & Amino acid derivatives & $329.84 \%$ & 1.19 & 4.28 & Up & $-51.52 \%$ & 1.56 & 0.48 & Down & $104.88 \%$ & 1.23 & 2.04 & Up \\
\hline L-Glutamic acid O-glucoside & Amino acid derivatives & $277.32 \%$ & 1.17 & 3.65 & Up & & & & - & $156.72 \%$ & 1.21 & 2.50 & Up \\
\hline 3-(2-Naphthyl)-D-alanine & Amino acid derivatives & & & & - & $-53.37 \%$ & 1.43 & 0.44 & Down & $-53.56 \%$ & 1.01 & 0.42 & Down \\
\hline Glutathione oxidized & Amino acid derivatives & & & & - & & & & - & $-57.12 \%$ & 1.23 & 0.43 & Down \\
\hline N-Acetyl-L-glutamic acid & Amino acid derivatives & $139.85 \%$ & 1.19 & 2.38 & Up & & & & - & $143.20 \%$ & 1.22 & 2.41 & Up \\
\hline $\begin{array}{l}\text { (-)-3-(3,4-Dihydroxyphenyl)-2- } \\
\text { methylalanine }\end{array}$ & Amino acid derivatives & $201.64 \%$ & 1.17 & 2.89 & Up & & & & - & $183.83 \%$ & 1.18 & 2.67 & Up \\
\hline Pyrrole-2-carboxylic acid & Amino acid derivatives & $666.89 \%$ & 1.20 & 7.55 & Up & & & & - & $879.38 \%$ & 1.23 & 9.62 & Up \\
\hline Phenylacetyl-L-glutamine & Amino acid derivatives & & & & - & & & & - & $197.73 \%$ & 1.10 & 2.71 & Up \\
\hline N-(3-Indolylacetyl)-L-alanine & Amino acid derivatives & & & & - & & & & - & $-48.02 \%$ & 1.11 & 0.50 & Down \\
\hline & & & & & & & & & & & & & (Continued \\
\hline
\end{tabular}


TABLE 2 | Continued

\begin{tabular}{|c|c|c|c|c|c|c|c|c|c|c|c|c|c|}
\hline \multirow[t]{2}{*}{ Chemical } & \multirow[t]{2}{*}{ Class } & \multicolumn{4}{|c|}{ Fried filling vs. raw filling } & \multicolumn{4}{|c|}{ Baked filling vs. fried filling } & \multicolumn{4}{|c|}{ Baked filling vs. raw filling } \\
\hline & & $\begin{array}{c}\text { Variation } \\
\text { percentage } \\
(\%)\end{array}$ & VIP & $\begin{array}{l}\text { Fold } \\
\text { change }\end{array}$ & $\begin{array}{l}\text { Up/Down } \\
\text { regulated }\end{array}$ & $\begin{array}{c}\text { Variation } \\
\text { percentage } \\
(\%)\end{array}$ & VIP & $\begin{array}{l}\text { Fold } \\
\text { change }\end{array}$ & $\begin{array}{l}\text { Up/Down } \\
\text { regulated }\end{array}$ & $\begin{array}{c}\text { Variation } \\
\text { percentage } \\
(\%)\end{array}$ & VIP & $\begin{array}{l}\text { Fold } \\
\text { change }\end{array}$ & $\begin{array}{l}\text { Up/Down } \\
\text { regulated }\end{array}$ \\
\hline Glutathione reduced form & Amino acid derivatives & $-66.52 \%$ & 1.12 & 0.30 & Down & & & & - & $-81.85 \%$ & 1.21 & 0.17 & Down \\
\hline $\mathrm{N}^{\prime}$-Formylkynurenine & Amino acid derivatives & & & & - & & & & - & $106.42 \%$ & 1.22 & 2.07 & Up \\
\hline L-Pipecolic acid & Amino acid derivatives & $148.41 \%$ & 1.20 & 2.47 & Up & & & & - & $191.76 \%$ & 1.24 & 2.90 & Up \\
\hline 3-N-Methyl-L-histidine & Amino acid derivatives & $-68.42 \%$ & 1.18 & 0.31 & Down & & & & - & $-53.13 \%$ & 1.15 & 0.45 & Down \\
\hline Phe-Phe & Amino acid derivatives & & & & - & & & & - & $-49.19 \%$ & 1.13 & 0.49 & Down \\
\hline S-(5'-Adenosyl)-L-methionine & Amino acid derivatives & $-99.31 \%$ & 1.04 & 0.01 & Down & $-66.43 \%$ & 1.18 & 0.01 & Down & $-100.00 \%$ & 1.24 & 0.00 & Down \\
\hline N-Phenylacetylglycine & Amino acid derivatives & & & & - & $436.94 \%$ & 1.59 & 5.48 & Up & $257.74 \%$ & 1.18 & 3.65 & Up \\
\hline Hexanoyl glycine & Amino acid derivatives & & & & - & & & & - & $208.35 \%$ & 1.02 & 2.59 & Up \\
\hline CYS-GLY & Amino acid derivatives & & & & - & + & 1.11 & 2.37 & Up & $-61.97 \%$ & 1.13 & 0.36 & Down \\
\hline $\mathrm{H}$-HomoArg-OH & Amino acid derivatives & & & & - & & & & - & $-52.09 \%$ & 1.18 & 0.47 & Down \\
\hline $\begin{array}{l}\text { 1,4-dihydro-1-Methyl-4-oxo-3- } \\
\text { pyridinecarboxamide }\end{array}$ & Pyridine derivatives & $-56.87 \%$ & 1.19 & 0.43 & Down & & & & - & & & & - \\
\hline Pantothenol & Alcohols and polyols & $-85.25 \%$ & 1.20 & 0.15 & Down & & & & - & $-88.42 \%$ & 1.24 & 0.12 & Down \\
\hline 1,5-Anhydro-D-glucitol & Alcohols and polyols & $1059.15 \%$ & 1.20 & 11.23 & Up & & & & - & $956.15 \%$ & 1.23 & 9.96 & Up \\
\hline sn-Glycero-3-phosphocholine & Cholines & & & & - & & & & - & $223.75 \%$ & 1.21 & 3.17 & Up \\
\hline $\mathrm{N}^{\prime}, \mathrm{N}^{\prime \prime}$-disinapoylspermidine & Phenolamides & & & & - & & & & - & $-59.34 \%$ & 1.17 & 0.40 & Down \\
\hline N-Feruloyl spermidine & Phenolamides & $-65.26 \%$ & 1.17 & 0.34 & Down & $-47.97 \%$ & 1.34 & 0.49 & Down & $-82.69 \%$ & 1.18 & 0.17 & Down \\
\hline Spermidine & Phenolamides & $1080.60 \%$ & 1.19 & 11.07 & Up & & & & - & $616.06 \%$ & 1.21 & 6.89 & Up \\
\hline Agmatine & Phenolamides & $390.76 \%$ & 1.19 & 4.72 & Up & & & & - & $315.40 \%$ & 1.08 & 3.80 & Up \\
\hline $\begin{array}{l}\mathrm{N} \text {-(4'-O-glycosyl)-p-coumaroyl } \\
\text { agmatine }\end{array}$ & Phenolamides & $-59.81 \%$ & 1.19 & 0.40 & Down & & & & - & $-75.60 \%$ & 1.24 & 0.24 & Down \\
\hline $\mathrm{N}^{\prime}$-Feruloyl putrescine & Phenolamides & & & & - & & & & - & $-99.99 \%$ & 1.24 & 0.00 & Down \\
\hline $\mathrm{N}$-Feruloyl putrescine & Phenolamides & $-65.83 \%$ & 1.09 & 0.34 & Down & & & & - & $-80.08 \%$ & 1.21 & 0.20 & Down \\
\hline N-Acetylputrescine & Phenolamides & $633.73 \%$ & 1.19 & 7.16 & Up & & & & - & $589.41 \%$ & 1.20 & 6.84 & Up \\
\hline N2-methylguanosine & $\begin{array}{l}\text { Nucleotide and its } \\
\text { derivatives }\end{array}$ & & & & - & & & & - & $-56.92 \%$ & 1.21 & 0.43 & Down \\
\hline Adenosine $3^{\prime}$-monophosphate & $\begin{array}{l}\text { Nucleotide and its } \\
\text { derivatives }\end{array}$ & $354.12 \%$ & 1.19 & 4.54 & Up & & & & - & $384.82 \%$ & 1.23 & 4.79 & Up \\
\hline
\end{tabular}


TABLE 2 | Continued

\begin{tabular}{|c|c|c|c|c|c|c|c|c|c|c|c|c|c|}
\hline \multirow[t]{2}{*}{ Chemical } & \multirow[t]{2}{*}{ Class } & \multicolumn{4}{|c|}{ Fried filling vs. raw filling } & \multicolumn{4}{|c|}{ Baked filling vs. fried filling } & \multicolumn{4}{|c|}{ Baked filling vs. raw filling } \\
\hline & & $\begin{array}{l}\text { Variation } \\
\text { percentage } \\
(\%)\end{array}$ & VIP & $\begin{array}{l}\text { Fold } \\
\text { change }\end{array}$ & $\begin{array}{l}\text { Up/Down } \\
\text { regulated }\end{array}$ & $\begin{array}{l}\text { Variation } \\
\text { percentage } \\
(\%)\end{array}$ & VIP & $\begin{array}{l}\text { Fold } \\
\text { change }\end{array}$ & $\begin{array}{l}\text { Up/Down } \\
\text { regulated }\end{array}$ & $\begin{array}{l}\text { Variation } \\
\text { percentage } \\
(\%)\end{array}$ & VIP & $\begin{array}{l}\text { Fold } \\
\text { change }\end{array}$ & $\begin{array}{l}\text { Up/Down } \\
\text { regulated }\end{array}$ \\
\hline $\begin{array}{l}\text { Nicotinic acid adenine } \\
\text { dinucleotide }\end{array}$ & $\begin{array}{l}\text { Nucleotide and its } \\
\text { derivatives }\end{array}$ & $-79.78 \%$ & 1.20 & 0.20 & Down & $-58.38 \%$ & 1.51 & 0.42 & Down & $-91.49 \%$ & 1.23 & 0.08 & Down \\
\hline Inosine 5'-monophosphate & $\begin{array}{l}\text { Nucleotide and its } \\
\text { derivatives }\end{array}$ & $307.27 \%$ & 1.18 & 4.01 & Up & & & & - & $302.25 \%$ & 1.20 & 3.86 & Up \\
\hline iP7G & $\begin{array}{l}\text { Nucleotide and its } \\
\text { derivatives }\end{array}$ & $454.83 \%$ & 1.19 & 5.41 & Up & & & & - & $959.51 \%$ & 1.23 & 10.60 & Up \\
\hline Adenosine 5'-monophosphate & $\begin{array}{l}\text { Nucleotide and its } \\
\text { derivatives }\end{array}$ & $260.29 \%$ & 1.19 & 3.59 & Up & & & & - & $280.91 \%$ & 1.22 & 3.77 & Up \\
\hline Guanosine 5'-monophosphate & $\begin{array}{l}\text { Nucleotide and its } \\
\text { derivatives }\end{array}$ & & & & - & & & & - & $150.43 \%$ & 1.15 & 2.51 & Up \\
\hline Uridine $5^{\prime}$-diphospho-D-glucose & $\begin{array}{l}\text { Nucleotide and its } \\
\text { derivatives }\end{array}$ & $-63.26 \%$ & 1.17 & 0.36 & Down & $-50.79 \%$ & 1.54 & 0.49 & Down & $-82.24 \%$ & 1.24 & 0.18 & Down \\
\hline $\begin{array}{l}\text { 2'-Deoxyinosine-5'- } \\
\text { monophosphate }\end{array}$ & $\begin{array}{l}\text { Nucleotide and its } \\
\text { derivatives }\end{array}$ & $-65.70 \%$ & 1.20 & 0.34 & Down & & & & - & $-80.44 \%$ & 1.22 & 0.19 & Down \\
\hline Adenosine O-ribose & $\begin{array}{l}\text { Nucleotide and its } \\
\text { derivatives }\end{array}$ & $-58.63 \%$ & 1.20 & 0.41 & Down & & & & - & $-52.89 \%$ & 1.16 & 0.47 & Down \\
\hline Uridine & $\begin{array}{l}\text { Nucleotide and its } \\
\text { derivatives }\end{array}$ & $-58.53 \%$ & 1.19 & 0.41 & Down & & & & - & & & & - \\
\hline Cytosine & $\begin{array}{l}\text { Nucleotide and its } \\
\text { derivatives }\end{array}$ & & & & - & $140.29 \%$ & 1.57 & 2.36 & Up & & & & - \\
\hline Adenine & $\begin{array}{l}\text { Nucleotide and its } \\
\text { derivatives }\end{array}$ & $-50.73 \%$ & 1.19 & 0.49 & Down & & & & - & & & & - \\
\hline$\beta$-Nicotinamide mononucleotide & $\begin{array}{l}\text { Nucleotide and its } \\
\text { derivatives }\end{array}$ & $-84.25 \%$ & 1.20 & 0.16 & Down & $-58.14 \%$ & 1.58 & 0.40 & Down & $-93.66 \%$ & 1.24 & 0.06 & Down \\
\hline 1-Methylxanthine & $\begin{array}{l}\text { Nucleotide and its } \\
\text { derivatives }\end{array}$ & + & 1.21 & 5315.81 & Up & & & & - & + & 1.24 & 4970.37 & Up \\
\hline Adenosine & $\begin{array}{l}\text { Nucleotide and its } \\
\text { derivatives }\end{array}$ & $107.54 \%$ & 1.20 & 2.07 & Up & & & & - & $158.06 \%$ & 1.10 & 2.60 & Up \\
\hline 5-Methyluridine & $\begin{array}{l}\text { Nucleotide and its } \\
\text { derivatives }\end{array}$ & + & 1.21 & 18098.52 & Up & & & & - & + & 1.24 & 20249.26 & Up \\
\hline Guanine & $\begin{array}{l}\text { Nucleotide and its } \\
\text { derivatives }\end{array}$ & & & & - & & & & - & $102.46 \%$ & 1.22 & 2.01 & Up \\
\hline Inosine & $\begin{array}{l}\text { Nucleotide and its } \\
\text { derivatives }\end{array}$ & & & & - & $108.31 \%$ & 1.54 & 2.03 & Up & & & & - \\
\hline
\end{tabular}


TABLE 2 | Continued

\begin{tabular}{|c|c|c|c|c|c|c|c|c|c|c|c|c|c|}
\hline \multirow[t]{2}{*}{ Chemical } & \multirow[t]{2}{*}{ Class } & \multicolumn{4}{|c|}{ Fried filling vs. raw filling } & \multicolumn{4}{|c|}{ Baked filling vs. fried filling } & \multicolumn{4}{|c|}{ Baked filling vs. raw filling } \\
\hline & & $\begin{array}{l}\text { Variation } \\
\text { percentage } \\
(\%)\end{array}$ & VIP & $\begin{array}{l}\text { Fold } \\
\text { change }\end{array}$ & $\begin{array}{l}\text { Up/Down } \\
\text { regulated }\end{array}$ & $\begin{array}{l}\text { Variation } \\
\text { percentage } \\
(\%)\end{array}$ & VIP & $\begin{array}{l}\text { Fold } \\
\text { change }\end{array}$ & $\begin{array}{l}\text { Up/Down } \\
\text { regulated }\end{array}$ & $\begin{array}{l}\text { Variation } \\
\text { percentage } \\
(\%)\end{array}$ & VIP & $\begin{array}{l}\text { Fold } \\
\text { change }\end{array}$ & $\begin{array}{l}\text { Up/Down } \\
\text { regulated }\end{array}$ \\
\hline Guanosine & Nucleotide and its derivates & $-55.49 \%$ & 1.20 & 0.45 & Down & & & & - & & & & - \\
\hline Deoxyguanosine & Nucleotide and its derivates & $-77.80 \%$ & 1.19 & 0.22 & Down & $677.30 \%$ & 1.57 & 7.20 & Up & & & & - \\
\hline $\begin{array}{l}\text { 2'-Deoxycytidine-5'- } \\
\text { monophosphate }\end{array}$ & Nucleotide and its derivates & + & 1.21 & 40852.96 & Up & & & & - & & & & - \\
\hline 1-Methyladenosine & Nucleotide and its derivates & $854.79 \%$ & 1.20 & 9.43 & Up & & & & - & $1488.22 \%$ & 1.24 & 15.92 & Up \\
\hline $\begin{array}{l}5^{\prime} \text {-Deoxy-5'- } \\
\text { (methylthio)adenosine }\end{array}$ & Nucleotide and its derivates & $276.54 \%$ & 1.20 & 3.77 & Up & & & & - & $242.45 \%$ & 1.23 & 3.43 & Up \\
\hline Guanosine monophosphate & Nucleotide and its derivates & $317.07 \%$ & 1.19 & 4.07 & Up & & & & - & $419.44 \%$ & 1.23 & 5.07 & Up \\
\hline 1,7-Dimethylxanthine & Nucleotide and its derivates & $-96.78 \%$ & 1.20 & 0.03 & Down & $163.97 \%$ & 1.41 & 2.37 & Up & $-92.39 \%$ & 1.23 & 0.08 & Down \\
\hline Cytidine 5'-monophosphate & Nucleotide and its derivates & $123.40 \%$ & 1.18 & 2.22 & Up & & & & - & $230.48 \%$ & 1.21 & 3.27 & Up \\
\hline Cyclic AMP & Nucleotide and its derivates & $-99.98 \%$ & 1.21 & 0.00 & Down & + & 1.63 & 38850.37 & Up & $503.61 \%$ & 1.14 & 6.28 & Up \\
\hline 8-Hydroxy-2-deoxyguanosine & Nucleotide and its derivates & & & & - & $188.52 \%$ & 1.24 & 2.96 & Up & & & & - \\
\hline Cytidine & Nucleotide and its derivates & & & & - & $155.64 \%$ & 1.55 & 2.58 & Up & $103.59 \%$ & 1.13 & 2.02 & Up \\
\hline $\begin{array}{l}\text { Guanosine } 3^{\prime}, 5^{\prime} \text {-cyclic } \\
\text { monophosphate }\end{array}$ & Nucleotide and its derivates & $2206.16 \%$ & 1.20 & 22.35 & Up & & & & - & $3051.38 \%$ & 1.24 & 29.88 & Up \\
\hline Deoxyadenosine & Nucleotide and its derivates & & & & - & $309.47 \%$ & 1.48 & 4.07 & Up & $598.04 \%$ & 1.16 & 7.21 & Up \\
\hline 2-(dimethylamino)guanosine & Nucleotide and its derivates & & & & - & $98.90 \%$ & 1.31 & 2.02 & Up & & & & - \\
\hline $\begin{array}{l}\text { Hypoxanthine-9- } \beta-D- \\
\text { arabinofuranoside }\end{array}$ & Nucleotide and its derivates & & & & - & $108.11 \%$ & 1.50 & 2.08 & Up & & & & - \\
\hline $\begin{array}{l}\mathrm{N} \text {-hexosyl-p-coumaroyl } \\
\text { serotonin }\end{array}$ & Tryptamine derivatives & $-64.78 \%$ & 1.19 & 0.35 & Down & & & & - & $-76.01 \%$ & 1.21 & 0.24 & Down \\
\hline N-Feruloyl serotonin & Tryptamine derivatives & $-54.01 \%$ & 1.05 & 0.41 & Down & & & & - & & & & - \\
\hline N-Feruloyl tryptamine & Tryptamine derivatives & $-65.74 \%$ & 1.20 & 0.34 & Down & & & & - & $-61.59 \%$ & 1.23 & 0.38 & Down \\
\hline
\end{tabular}


TABLE 2 | Continued

\begin{tabular}{|c|c|c|c|c|c|c|c|c|c|c|c|c|c|}
\hline \multirow[t]{2}{*}{ Chemical } & \multirow[t]{2}{*}{ Class } & \multicolumn{4}{|c|}{ Fried filling vs. raw filling } & \multicolumn{4}{|c|}{ Baked filling vs. fried filling } & \multicolumn{4}{|c|}{ Baked filling vs. raw filling } \\
\hline & & $\begin{array}{l}\text { Variation } \\
\text { percentage } \\
(\%)\end{array}$ & VIP & $\begin{array}{l}\text { Fold } \\
\text { change }\end{array}$ & $\begin{array}{l}\text { Up/Down } \\
\text { regulated }\end{array}$ & $\begin{array}{l}\text { Variation } \\
\text { percentage } \\
(\%)\end{array}$ & VIP & $\begin{array}{l}\text { Fold } \\
\text { change }\end{array}$ & $\begin{array}{l}\text { Up/Down } \\
\text { regulated }\end{array}$ & $\begin{array}{c}\text { Variation } \\
\text { percentage } \\
(\%)\end{array}$ & VIP & $\begin{array}{l}\text { Fold } \\
\text { change }\end{array}$ & $\begin{array}{l}\text { Up/Down } \\
\text { regulated }\end{array}$ \\
\hline Camptothecin & Alkaloids & $115.81 \%$ & 1.14 & 2.09 & Up & & & & - & $150.83 \%$ & 1.15 & 2.37 & Up \\
\hline Betaine & Alkaloids & $211.70 \%$ & 1.20 & 3.09 & Up & $602.30 \%$ & 1.61 & 7.03 & Up & $2121.08 \%$ & 1.24 & 21.72 & Up \\
\hline Phytocassane D & Terpenoids & $-71.27 \%$ & 1.18 & 0.28 & Down & & & & - & $-65.36 \%$ & 1.09 & 0.36 & Down \\
\hline Limonin & Terpenoids & + & 1.21 & 1666.70 & Up & & & & - & + & 1.24 & 2001.59 & Up \\
\hline Nicotinate ribonucleoside & Nicotinic acid derivatives & & & & - & & & & - & $-56.92 \%$ & 1.23 & 0.43 & Down \\
\hline Nicotinic acid & Nicotinic acid derivatives & $117.40 \%$ & 1.20 & 2.17 & Up & & & & - & $170.28 \%$ & 1.23 & 2.70 & Up \\
\hline $\begin{array}{l}\text { 5-methoxyindole-3- } \\
\text { carbaldehyde }\end{array}$ & Indole derivatives & $-71.95 \%$ & 1.13 & 0.27 & Down & & & & - & & & & - \\
\hline Indole-5-carboxylic acid & Indole derivatives & & & & - & & & & - & $163.20 \%$ & 1.16 & 2.52 & Up \\
\hline 5-Hydroxyindole-3-acetic acid & Indole derivatives & & & & - & & & & - & $-56.11 \%$ & 1.23 & 0.44 & Down \\
\hline Methylglutaric acid & Organic acids & $-65.52 \%$ & 1.19 & 0.34 & Down & $-51.92 \%$ & 1.51 & 0.47 & Down & $-83.88 \%$ & 1.23 & 0.16 & Down \\
\hline 2-Aminoethanesulfinic acid & Organic acids & $470.39 \%$ & 1.14 & 4.89 & Up & & & & - & $695.50 \%$ & 1.18 & 6.59 & Up \\
\hline 2-Furoic acid & Organic acids & + & 1.21 & 841940.74 & Up & & & & - & + & 1.24 & 1084903.70 & Up \\
\hline Glutaric acid & Organic acids & $133.63 \%$ & 1.20 & 2.33 & Up & & & & - & $166.27 \%$ & 1.24 & 2.66 & Up \\
\hline 4-Oxopentanoate & Organic acids & $214.66 \%$ & 1.03 & 2.79 & Up & & & & - & $168.42 \%$ & 1.11 & 2.41 & Up \\
\hline Terephthalic acid & Organic acids & & & & - & & & & - & $136.19 \%$ & 1.19 & 2.33 & Up \\
\hline 2-Picolinic acid & Organic acids & $208.13 \%$ & 1.20 & 3.08 & Up & & & & - & $309.17 \%$ & 1.24 & 4.09 & Up \\
\hline Homogentisic acid & Organic acids & $1160.72 \%$ & 1.20 & 12.56 & Up & & & & - & $1485.63 \%$ & 1.24 & 15.76 & Up \\
\hline D-Pantothenic acid & Organic acids & $-65.31 \%$ & 1.18 & 0.34 & Down & & & & - & & & & - \\
\hline 3-Hydroxy-3-methyl butyric acid & Organic acids & $130.04 \%$ & 1.16 & 2.24 & Up & & & & - & $137.70 \%$ & 1.20 & 2.33 & Up \\
\hline D-Erythronolactone & Organic acids & $185.54 \%$ & 1.17 & 2.78 & Up & $106.46 \%$ & 1.52 & 2.06 & Up & $475.33 \%$ & 1.22 & 5.74 & Up \\
\hline Creatine & Organic acids & & & & - & $1146.83 \%$ & 1.61 & 12.45 & Up & $678.39 \%$ & 1.21 & 7.80 & Up \\
\hline 2-Aminoethanesulfonic acid & Organic acids & $578.57 \%$ & 1.20 & 6.73 & Up & & & & - & $1188.64 \%$ & 1.23 & 12.97 & Up \\
\hline Suberic acid & Organic acids & $197.14 \%$ & 1.14 & 2.85 & Up & & & & - & $177.00 \%$ & 1.18 & 2.65 & Up \\
\hline Citraconic acid & Organic acids & + & 1.21 & 231022.22 & Up & & & & - & + & 1.24 & 256744.44 & Up \\
\hline
\end{tabular}


TABLE 2 | Continued

\begin{tabular}{|c|c|c|c|c|c|c|c|c|c|c|c|c|c|}
\hline \multirow[t]{2}{*}{ Chemical } & \multirow[t]{2}{*}{ Class } & \multicolumn{4}{|c|}{ Fried filling vs. raw filling } & \multicolumn{4}{|c|}{ Baked filling vs. fried filling } & \multicolumn{4}{|c|}{ Baked filling vs. raw filling } \\
\hline & & $\begin{array}{c}\text { Variation } \\
\text { percentage } \\
(\%)\end{array}$ & VIP & $\begin{array}{l}\text { Fold } \\
\text { change }\end{array}$ & $\begin{array}{l}\text { Up/Down } \\
\text { regulated }\end{array}$ & $\begin{array}{c}\text { Variation } \\
\text { percentage } \\
(\%)\end{array}$ & VIP & $\begin{array}{l}\text { Fold } \\
\text { change }\end{array}$ & $\begin{array}{l}\text { Up/Down } \\
\text { regulated }\end{array}$ & $\begin{array}{c}\text { Variation } \\
\text { percentage } \\
(\%)\end{array}$ & VIP & $\begin{array}{l}\text { Fold } \\
\text { change }\end{array}$ & $\begin{array}{l}\text { Up/Down } \\
\text { regulated }\end{array}$ \\
\hline Mandelic acid & Organic acids & & & & - & & & & - & $117.32 \%$ & 1.06 & 2.20 & Up \\
\hline A-Ketoglutaric acid & Organic acids & $114.87 \%$ & 1.20 & 2.15 & Up & & & & - & $126.45 \%$ & 1.22 & 2.26 & Up \\
\hline $\begin{array}{l}\text { 3,4-Dihydroxybenzeneacetic } \\
\text { acid }\end{array}$ & Organic acids & $943.16 \%$ & 1.19 & 9.73 & Up & & & & - & $1275.36 \%$ & 1.23 & 12.81 & Up \\
\hline ethylmalonate & Organic acids & $106.55 \%$ & 1.14 & 2.00 & Up & & & & - & $144.32 \%$ & 1.19 & 2.42 & Up \\
\hline (Rs)-Mevalonic acid & Organic acids & $1509.63 \%$ & 1.18 & 14.18 & Up & & & & - & $2493.85 \%$ & 1.23 & 22.74 & Up \\
\hline trans,trans-Muconic acid & Organic acids & $261.50 \%$ & 1.18 & 3.50 & Up & & & & - & $473.77 \%$ & 1.23 & 5.59 & Up \\
\hline LysoPC 16:1 & Lipids_Glycerophospholipids & s $160.83 \%$ & 1.13 & 2.45 & Up & & & & - & $206.03 \%$ & 1.20 & 2.94 & Up \\
\hline LysoPC 18:2 & Lipids_Glycerophospholipids & s $202.86 \%$ & 1.14 & 2.93 & Up & $122.13 \%$ & 1.42 & 2.13 & Up & $538.39 \%$ & 1.22 & 6.24 & Up \\
\hline LysoPC 18:3 & Lipids_Glycerophospholipids & s $236.76 \%$ & 1.04 & 3.03 & Up & $123.49 \%$ & 1.19 & 2.11 & Up & $578.88 \%$ & 1.18 & 6.40 & Up \\
\hline LysoPC 16:0 & Lipids_Glycerophospholipids & s $196.70 \%$ & 1.11 & 2.86 & Up & & & & - & $406.37 \%$ & 1.20 & 5.03 & Up \\
\hline LysoPC 18:1 (2n isomer) & Lipids_Glycerophospholipids & & & & - & $192.53 \%$ & 1.52 & 2.85 & Up & $192.96 \%$ & 1.20 & 2.90 & Up \\
\hline LysoPC 18:3 (2n isomer) & Lipids_Glycerophospholipids & & & & - & & & & - & $122.54 \%$ & 1.16 & 2.13 & Up \\
\hline LysoPC 14:0 & Lipids_Glycerophospholipids & & & & - & $341.02 \%$ & 1.57 & 4.35 & Up & $201.51 \%$ & 1.16 & 2.92 & Up \\
\hline LysoPE 18:2 (2n isomer) & Lipids_Glycerophospholipids & & & & - & & & & - & $253.95 \%$ & 1.18 & 3.46 & Up \\
\hline LysoPE 18:0 ( $2 n$ isomer) & Lipids_Glycerophospholipids & s $226.78 \%$ & 1.03 & 2.84 & Up & $220.96 \%$ & 1.43 & 2.96 & Up & $765.64 \%$ & 1.19 & 8.42 & Up \\
\hline LysoPE 18:2 & Lipids_Glycerophospholipids & $s-52.50 \%$ & 1.16 & 0.47 & Down & $165.92 \%$ & 1.45 & 2.64 & Up & & & & - \\
\hline LysoPC 18:1 & Lipids_Glycerophospholipids & & & & - & & & & - & $280.22 \%$ & 1.15 & 3.58 & Up \\
\hline LysoPE 18:0 & Lipids_Glycerophospholipids & s- $-91.76 \%$ & 1.16 & 0.07 & Down & $1511.34 \%$ & 1.59 & 15.50 & Up & & & & - \\
\hline LysoPC 10:0 & Lipids_Glycerophospholipids & s-55.99\% & 1.05 & 0.40 & Down & $332.05 \%$ & 1.50 & 3.97 & Up & & & & - \\
\hline LysoPC 19:0 & Lipids_Glycerophospholipids & s $187.22 \%$ & 1.11 & 2.79 & Up & $597.01 \%$ & 1.59 & 6.54 & Up & $1900.05 \%$ & 1.23 & 18.22 & Up \\
\hline LysoPC 15:1 & Lipids_Glycerophospholipids & & & & - & $222.20 \%$ & 1.52 & 3.09 & Up & $232.09 \%$ & 1.18 & 3.23 & Up \\
\hline LysoPC 15:0 & Lipids_Glycerophospholipids & & & & - & $275.37 \%$ & 1.56 & 3.77 & Up & $217.58 \%$ & 1.16 & 3.14 & Up \\
\hline PC 19:2/16:0 & Lipids_Glycerophospholipids & & & & - & $431.85 \%$ & 1.25 & 3.50 & Up & $59007.31 \%$ & 1.07 & 58.69 & Up \\
\hline LysoPC 18:0 (2n isomer) & Lipids_Glycerophospholipids & & & & - & $197.08 \%$ & 1.47 & 2.98 & Up & $181.91 \%$ & 1.12 & 2.71 & Up \\
\hline LysoPC 17:0 & Lipids_Glycerophospholipids & & & & - & $165.57 \%$ & 1.38 & 2.72 & Up & & & & - \\
\hline LysoPE 18:1 & Lipids_Glycerophospholipids & s $-80.13 \%$ & 1.17 & 0.20 & Down & $134.47 \%$ & 1.52 & 2.23 & Up & $-54.06 \%$ & 1.16 & 0.45 & Down \\
\hline
\end{tabular}

(Continued) 
TABLE 2 | Continued

Chemical Class

Fried filling vs. raw filling

Baked filling vs. fried filling

Baked filling vs. raw filling

\begin{tabular}{|c|c|c|c|c|c|c|c|c|c|c|c|c|c|}
\hline & & & & & & & & & & & & & \\
\hline & & $\begin{array}{l}\text { Variation } \\
\text { percentage } \\
(\%)\end{array}$ & VIP & $\begin{array}{l}\text { Fold } \\
\text { change }\end{array}$ & $\begin{array}{l}\text { Up/Down } \\
\text { regulated }\end{array}$ & $\begin{array}{c}\text { Variation } \\
\text { percentage } \\
(\%)\end{array}$ & VIP & $\begin{array}{l}\text { Fold } \\
\text { change }\end{array}$ & $\begin{array}{l}\text { Up/Down } \\
\text { regulated }\end{array}$ & $\begin{array}{l}\text { Variation } \\
\text { percentage } \\
(\%)\end{array}$ & VIP & $\begin{array}{c}\text { Fold } \\
\text { change }\end{array}$ & $\begin{array}{l}\text { Up/Down } \\
\text { regulated }\end{array}$ \\
\hline LysoPE 18:1 (2n isomer) & Lipids_Glycerophospholipids & $-62.34 \%$ & 1.17 & 0.37 & Down & $291.54 \%$ & 1.61 & 3.80 & Up & & & & - \\
\hline LysoPC 20:4 & Lipids_Glycerophospholipids & & & & - & $232.49 \%$ & 1.50 & 3.27 & Up & $238.49 \%$ & 1.17 & 3.24 & Up \\
\hline LysoPC 14:0 (2n isomer) & Lipids_Glycerophospholipids & $-53.71 \%$ & 1.12 & 0.46 & Down & $269.23 \%$ & 1.56 & 3.48 & Up & & & & - \\
\hline LysoPC 16:0 (2n isomer) & Lipids_Glycerophospholipids & $295.77 \%$ & 1.15 & 3.82 & Up & $127.99 \%$ & 1.38 & 2.18 & Up & $745.08 \%$ & 1.22 & 8.32 & Up \\
\hline LysoPC 18:0 & Lipids_Glycerophospholipids & & & & - & $401.07 \%$ & 1.56 & 5.04 & Up & $521.49 \%$ & 1.18 & 5.80 & Up \\
\hline LysoPC 20:1 (2n isomer) & Lipids_Glycerophospholipids & & & & - & $128.93 \%$ & 1.53 & 2.30 & Up & & & & - \\
\hline LysoPC 20:1 & Lipids_Glycerophospholipids & & & & - & $129.41 \%$ & 1.53 & 2.31 & Up & & & & - \\
\hline LysoPE 14:0 (2n isomer) & Lipids_Glycerophospholipids & & & & - & $139.83 \%$ & 1.55 & 2.26 & Up & & & & - \\
\hline LysoPE 16:0 (2n isomer) & Lipids_Glycerophospholipids & $185.01 \%$ & 1.10 & 2.77 & Up & & & & - & $419.60 \%$ & 1.22 & 5.19 & Up \\
\hline DGMG (18:2) isomer1 & Lipids_Glycerolipids & & & & - & $192.32 \%$ & 1.12 & 3.02 & Up & & & & - \\
\hline DGMG (18:2) isomer3 & Lipids_Glycerolipids & & & & - & $420.39 \%$ & 1.48 & 5.14 & Up & $683.96 \%$ & 1.18 & 7.44 & Up \\
\hline MAG (18:2) isomer1 & Lipids_Glycerolipids & & & & - & $440.62 \%$ & 1.47 & 5.60 & Up & $597.46 \%$ & 1.15 & 6.26 & Up \\
\hline MAG (18:4) isomer2 & Lipids_Glycerolipids & & & & - & $157.95 \%$ & 1.46 & 2.48 & Up & & & & - \\
\hline MAG (18:1) isomer2 & Lipids_Glycerolipids & & & & - & $191.58 \%$ & 1.18 & 2.97 & Up & & & & - \\
\hline MAG (18:2) & Lipids_Glycerolipids & $-56.04 \%$ & 1.08 & 0.40 & Down & & & & - & & & & - \\
\hline MAG (18:3) isomer3 & Lipids_Glycerolipids & & & & - & $201.68 \%$ & 1.13 & 2.87 & Up & $320.09 \%$ & 1.03 & 4.11 & Up \\
\hline MGMG (18:2) isomer1 & Lipids_Glycerolipids & & & & - & $994.36 \%$ & 1.60 & 11.05 & Up & $1361.25 \%$ & 1.23 & 14.08 & Up \\
\hline MAG (18:3) isomer4 & Lipids_Glycerolipids & $-50.80 \%$ & 1.04 & 0.44 & Down & & & & - & & & & - \\
\hline MAG (18:3) isomer2 & Lipids_Glycerolipids & & & & - & $-77.99 \%$ & 1.20 & 0.23 & Down & & & & - \\
\hline MAG (18:1) isomer1 & Lipids_Glycerolipids & $-70.79 \%$ & 1.17 & 0.28 & Down & & & & - & & & & - \\
\hline MGMG (18:2) isomer2 & Lipids_Glycerolipids & & & & - & $219.11 \%$ & 1.29 & 2.64 & Up & $207.38 \%$ & 1.11 & 2.91 & Up \\
\hline MAG (18:3) isomer1 & Lipids_Glycerolipids & & & & - & $121.22 \%$ & 1.12 & 2.23 & Up & & & & - \\
\hline 14,15-Dehydrocrepenynic acid & Lipids_Fatty acids & $-93.89 \%$ & 1.20 & 0.06 & Down & & & & - & $-92.86 \%$ & 1.24 & 0.07 & Down \\
\hline delta-Tridecalactone & Lipids_Fatty acids & + & 1.21 & 5796.89 & Up & & & & - & + & 1.24 & 6310.04 & Up \\
\hline Punicic acid & Lipids_Fatty acids & $-76.28 \%$ & 1.16 & 0.23 & Down & & & & - & $-67.90 \%$ & 1.18 & 0.32 & Down \\
\hline 9,10-EODE & Lipids_Fatty acids & $-71.04 \%$ & 1.09 & 0.30 & Down & & & & - & $-56.12 \%$ & 1.18 & 0.43 & Down \\
\hline 9-HOTrE & Lipids_Fatty acids & $-78.29 \%$ & 1.15 & 0.22 & Down & & & & - & $-75.34 \%$ & 1.22 & 0.24 & Down \\
\hline
\end{tabular}

(Continued) 


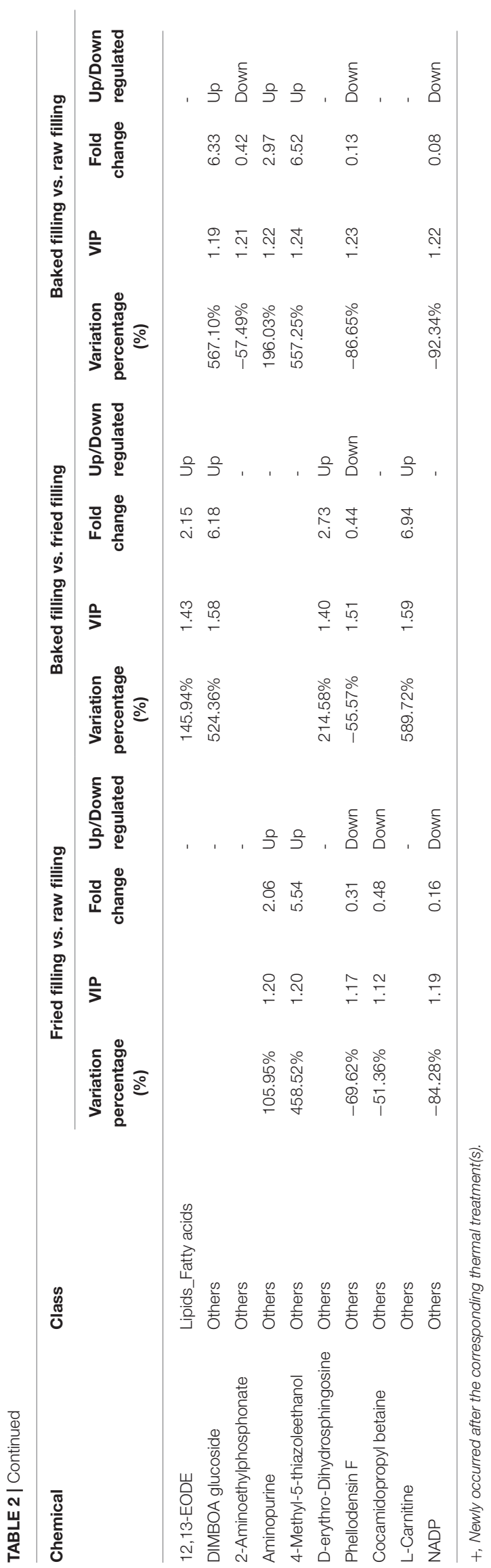

by 2.5 times, whereas some of its glycosides, such as quercetin3,4'-O-diglucoside, quercetin 7-O-malonylhexosyl-hexoside, and quercetin 3-arabinoside, decreased remarkably after stir-frying. Kaempferol glycosides, such as kaempferol 3-O-galactoside, kaempferol 3-O-rutinoside, kaempferol 3-O-robinobioside, kaempferol 3,7-dirhamnoside, and kaempferol-3-O-robinoside7-O-rhamnoside, decreased after thermal processing, whereas the aglycone kaempferol increased. The decreasing trends of the flavonol glycosides could be attributed to the deglycosylation that occurred during thermal processing as discussed in our previous study (8).

Changes in other phenolic compounds in blueberry filling during pastry preparation can be also determined by the widely targeted metabolomic analysis with UHPLC-MS/MS. For example, protocatechuic acid in the blueberry filling increased by four times after stir-frying. Protocatechuic acid was reported to be thermally degraded from cyanidin by the liberation of the catecholic B-ring or from flavonoids such as quercetin (9). It shared the same ortho-dihydroxyphenyl chemical moiety with cyanidin, which was reported to be critical for the biological properties of anthocyanins $(22,23)$. Thus, the degradation of cyanidin to protocatechuic acid might not result in the reduction of bioactivity. Protocatechuic acid was demonstrated to possess chemopreventive activity against several different types of cancers in animal and cell studies $(24,25)$. Coumarins are another group of phenolic compounds that possess various biological and therapeutic properties such as anti-oxidant, anti-microbial, anti-viral, antidiabetic, anti-coagulant, estrogenic, vasodilator, anti-convulsant, anti-inflammatory, anti-hypertensive, and anti-cancer activities (26). In blueberry fillings, the amount of 6-methoxy-7,8dihydroxycoumarin increased remarkably by 13 times after stirfrying, and by 34 times by the end of thermal processing. The degradation of anthocyanins might contribute to the increase (27). Moreover, 4-hydroxybenzoic acid was also reported to be the thermal degradation product of anthocyanin pelargonidin-3glucoside (28). Although its content did not increase significantly in the filling after stir-frying, the abundance of pelargonidin-3glucoside decreased remarkably. However, the final abundance of the compound increased significantly in the baked product compared with the raw filling. Other benzoic acid derivatives, such as gallic acid, vanillin, syringaldehyde, methyl gallate, 2,5-dihydroxybenzoic acid, 4-hydroxybenzaldehyde, anthranilic acid, and 2,3-dihydroxybenzoic acid, showed elevations in the filling after stir-frying. The increases in the amounts of vanillin, syringaldehyde, phenol, and phenol derivatives during heat treatment might be attributed to the thermal degradation of lignin (29). The thermal decomposition of the lignin polymer commonly started with the cleavage of the $\alpha$-ether and $\beta$ ether bonds. As a result, a mixture of phenol-, guaiacyl-, and syringyl-type derivatives with their substituents in the aromatic ring was released (30); the attack of oxygen might lead to the formation of aromatic aldehydes and ketones (31). Amongst the hydroxycinnamoyl derivatives detected, 12 varied under the stir-frying treatment. Ferulic acid, cinnamic acid, p-coumaric acid, and sinapic acid showed increasing trends similar to that of benzoic acid derivatives. Moreover, the catechin 
derivatives, protocatechuic acid, protocatechuic aldehyde, and epigallocatechin showed increasing trends during stir-frying. The increases in these phenolic acids might be explained by the thermal degradation of flavonoids and the release of bound phenolic compounds from the insoluble polymers in the fruits under heat treatments $(32,33)$.

Amongst the flavanones, the abundance of naringenin and its chalcone increased after stir-frying, whereas that of naringenin $O$-malonylhexoside decreased. Lou et al. (34) observed increases in the amounts of naringenin, tangeretin, and gallic acid in the immature calamondin after heat treatment at $150^{\circ} \mathrm{C}$ for $1.5 \mathrm{~h}$ and attributed these increases to the liberation of the compound from the immature calamondin peel during heating. Heat treatment could cause the degradation of cell wall structure to release the bound phenolic acids. Accordingly, the contents of tangeretin and gallic acid in the blueberry filling also increased after stir-frying (Table 1). However, none of the flavanones displayed notable changes in fillings during baking. Interestingly, sakuranetin, which is a methylation product of naringenin and has not been detected in the raw fillings, was produced in fried and baked fillings. Although the biosynthesis of sakuranetin from naringenin in plants (35) and the reversable $O$-demethylation of sakuranetin to naringenin in living tissues under the presence of cytochrome P450 monooxygenases have been well-studied (36), the formation of these components and their interactions and correlations are difficult to speculate without further investigations.

Flavone C-glycosides displayed no variation during baking; during stir-frying, seven flavone C-glycosides showed significant decreases in abundance (Table 1). By contrast, two flavone C-glycosides, namely, 8-C-hexosyl chrysoeriol $O$-hexoside and hesperetin $\mathrm{C}$-hexoside $\mathrm{O}$-hexoside, increased significantly in the fillings during stir-frying. Moreover, 8-C-hexosyl chrysoeriol $O$-hexoside was only observed after stir-frying. Therefore, it is a novel degradation product that mostly originated from flavonoids. Amongst the flavones, tricin 5-O-hexosyl-O-hexoside and tricin $O$-hexosyl- $O$-syringin alcohol were novel degradation products that appeared after stir-frying. The other flavones either decreased or remained constant during stir-frying.

\section{Variation in Other Chemicals in Blueberry Fillings During Thermal Processing}

Other than flavonoids, the other chemicals in the blueberry fillings were also fully investigated by the widely targeted metabolomic analysis with UHPLC-MS/MS to obtain a comprehensive view on the variation in whole chemical compounds in blueberry filling during pastry preparation and gain further insight into the underlying mechanisms.

During thermal processing, the abundance of sucrose decreased by $32 \%$ at the stage of stir-frying, while the amount of glucose remained unchanged after stir-frying. The contents of the oxidized products of glucose, gluconic acid and glucono-1,5-lactone, increased by 52 and 123\%, respectively (Table 2). However, only the variation in glucono-1,5-lactone was statistically significant. The increases in the amount of gluconic acid and glucono-1,5-lactone might be attributed to the oxidation of glucose induced by $\mathrm{H}_{2} \mathrm{O}_{2}$ in presence of ferrous ion (37), in which $\mathrm{H}_{2} \mathrm{O}_{2}$ can be produced from ascorbic acid catalyzed by $\mathrm{Cu}(\mathrm{II})$ (38).

Amongst all the 28 amino acids detected, only L-methionine, L-glutamine, and L- $(+)$-ornithine decreased significantly in the blueberry filling after stir-frying. Baking only affected the amount of L-glutamine. In comparison with the raw filling, baked filling showed higher levels of L-histidine, L- $(+)$-arginine, Lmethionine, and L-glutamine. Pyrrole-2-carboxylic acid, which is a product of the Maillard browning reaction between xylose and amino acid/peptides, increased sharply by $667 \%$ after stir-frying $(39,40)$.

Fang et al. (41) found that triphosphates and diphosphates of nucleotides could be degraded into monophosphate analogs or even nucleosides during heating. This finding might explain the considerable increases in the amounts of nucleosides and their monophosphates in blueberry fillings after heating. The abundance of adenosine, adenosine $3^{\prime}$-monophosphate, inosine $5^{\prime}$-monophosphate, adenosine $5^{\prime}$-monophosphate, guanosine monophosphate, cytidine $5^{\prime}$-monophosphate, and guanosine $3^{\prime}, 5^{\prime}$-cyclic monophosphate increased by 108 , $354,307,260,317,123$, and 2206\%, respectively, after stirfrying. $2^{\prime}$-Deoxycytidine- $5^{\prime}$-monophosphate appeared as a new product in the filling after stir-frying. Surprisingly, the degradation products of methylated nucleosides extensively increased; for instance, 1 -methyladenosine increased by $855 \%$, and 5-methyluridine and 1-methylxanthine were produced only after the thermal treatment of stir-frying. The results demonstrated that nucleosides and even free nucleotides would be degraded during heat treatment (41). Moreover, the thermal processing, especially at acidic conditions, might even elicit DNA degradation. Bitskinashvili et al. (42) reported that the combined thermal-acid treatment at $100^{\circ} \mathrm{C}$ and $\mathrm{pH}$ 2-4 could cause considerable degradations of maize and wheat DNA.

Amongst the seven alkaloids, only two showed significant variation after thermal processing, and both displayed increasing trends. However, three tryptamine derivatives were detected to be significantly different and showed reducing trends under the thermal treatment of stir-frying. The other components, such as organic acids, lipids, and amino acid derivatives, showed individually different variation behaviors under thermal processing (Table 2). Amongst the organic acids, only methylglutaric and D-pantothenic acids decreased in terms of abundance upon thermal treatments. Citraconic acid and 2-furoic acid occurred as new products after the thermal treatment of stir-frying. Glutaric acid, which is a degradation product that originated from sugar and lignin under heat treatment, increased by 1.3 times in the filling after stir-frying. D-Erythronolactone, which is a lactone formed by the reaction of D-xylose and calcium hydroxide (43), increased by 186 and 106\%, respectively, after stir-frying and baking. Butter was incorporated into the pastry fillings and exposed to high temperature during stir-frying. Heating of butter increased its lactone content (43). As a result, the tridecalactone, which is a component in butter, was observed after stir-frying. 


\section{CONCLUSIONS}

In this study, we applied a widely targeted metabolomic approach to investigate the global chemical changes during different thermal processing procedures, namely, stir-frying and baking, which were involved in the preparation of traditional blueberry filled pastry. A total of 630 chemicals were detected in blueberry fillings, and 288 were screened to be differential chemicals as samples underwent different thermal treatments. In comparison with baking, stir-frying contributed the most to the deviation of the abundance of components in blueberry fillings. Anthocyanin was the most sensitive toward thermal treatments. The other classes of phytochemicals, such as glycerophospholipids, nucleotides and their derivatives, benzoic acid derivatives, flavonols, flavones, flavanones, flavone C-glycosides, hydroxycinnamoyl derivatives, and phenolamides, were also comparably sensitive toward thermal processing. The variation in the chemicals and the novel compounds that appeared after thermal processing detected by this widely targeted metabolomic analysis could provide important insights into the degradation mechanism of phytochemicals and interactions between food ingredients. However, metabolomic analysis for the total understanding of the variation in chemical features and underlying mechanisms during food preparation and corresponding reactions remains a major challenge. The currently developed metabolomic databases are mostly specific to food products, such as FooDB, which is a subset of the Human Metabolite Database (44). This database contains information about thousands of phytochemicals but lacks information on the corresponding chemical degradation products or interaction products. Additional efforts should be applied on the development of metabolomic approaches and their application in investigating food processing.

\section{REFERENCES}

1. Grace MH, Xiong J, Esposito D, Ehlenfeldt M, Lila MA. Simultaneous LCMS quantification of anthocyanins and non-anthocyanin phenolics from blueberries with widely divergent profiles and biological activities. Food Chem. (2019) 227:336-46. doi: 10.1016/j.foodchem.2018.10.101

2. Nadulski R, Maslowski A, Mazurek A, Sobczak P, Szmigielski M, Zukiewicz-Sobczak W, et al. Vitamin $\mathrm{C}$ and lutein content of northern highbush blueberry (Vaccinium corymbosum L.) juice processed using freezing and thawing. J Food Meas Charact. (2019) 13:2521-8. doi: 10.1007/s11694-019-00172-x

3. Nemzer B, Vargas L, Xia XY, Sintara M, Feng H. Phytochemical and physical properties of blueberries, tart cherries, strawberries, and cranberries as affected by different drying methods. Food Chem. (2018) 262:24250. doi: 10.1016/j.foodchem.2018.04.047

4. Cesa S, Carradori S, Bellagamba G, Locatelli M, Casadei MA, Masci A, et al. Evaluation of processing effects on anthocyanin content and colour modifications of blueberry (Vaccinium spp.) extracts: comparison between HPLC-DAD and CIELAB analyses. Food Chem. (2017) 232:11423. doi: 10.1016/j.foodchem.2017.03.153

5. Klopsch R, Baldermann S, Voss A, Rohn S, Schreiner M, Neugart S. Bread enriched with legume microgreens and leaves-ontogenetic and baking-driven changes in the profile of secondary plant metabolites. Front Chem. (2018) 6:322. doi: $10.3389 /$ fchem. 2018.00322

\section{DATA AVAILABILITY STATEMENT}

The original contributions presented in the study are included in the article/Supplementary Material, further inquiries can be directed to the corresponding author/s.

\section{AUTHOR CONTRIBUTIONS}

JZ, WH, SO, and PL contributed conception and design of the study. JZ, ZW, NY, and $\mathrm{KZ}$ performed the experiments. $\mathrm{JZ}, \mathrm{ZW}$, and $\mathrm{PL}$ organized the database and performed the statistical analysis. JZ and SO wrote the first draft of the manuscript. PL and $\mathrm{ZW}$ revised the manuscript. All authors contributed to the article and approved the submitted version.

\section{FUNDING}

This work was supported by the National Natural Science Foundation of China [Nos. 31701607 and 31972180]; the Pearl River Talent Program (No. 20170096) and the Science and Technology Planning Project (Grant No. 2018B050502008) by Guangdong Science and Technology Department, Guangdong, China; and the Open Fund of Key Laboratory of Biotechnology and Bioresources Utilization (Dalian Minzu University), Ministry of Education (No. KF2018003), China.

\section{SUPPLEMENTARY MATERIAL}

The Supplementary Material for this article can be found online at: https://www.frontiersin.org/articles/10.3389/fnut.2020. 569172/full\#supplementary-material

6. Zhao Y, Wu XL, Yu LL, Chen P. Retention of polyphenols in blueberries (Vaccinium corymbosum) after different cooking methods, using UHPLCDAD-MS based metabolomics. J Food Compos Anal. (2017) 56:5566. doi: 10.1016/j.jfca.2016.12.003

7. Hager A, Howard LR, Prior RL, Brownmiller C. Processing and storage effects on monomeric anthocyanins, percent polymeric color, and antioxidant capacity of processed black raspberry products. J Food Sci. (2008) 73:H134H140. doi: 10.1111/j.1750-3841.2008.00855.x

8. Yang N, Qiu RX, Yang S, Zhou KN, Wang CT, Ou SY, et al. Influences of stir-frying and baking on flavonoid profile, antioxidant property, and hydroxymethylfurfural formation during preparation of blueberry-filled pastries. Food Chem. (2019) 287:167-75. doi: 10.1016/j.foodchem.2019.02.053

9. Teegarden MD, Schwartz SJ, Cooperstone JL. Profiling the impact of thermal processing on black raspberry phytochemicals using untargeted metabolomics. Food Chem. (2019) 274:7828. doi: 10.1016/j.foodchem.2018.09.053

10. Pérez-Míguez R, Sánchez-López E, Plaza M, Castro-Puyana M, Marina ML. A non-targeted metabolomic approach based on reversed-phase liquid chromatography-mass spectrometry to evaluate coffee roasting process. Anal Bioanal Chem. (2018) 410:7859-70. doi: 10.1007/s00216-018-1405-Z

11. Nikolantonaki M, Julien P, Coelho C, Roullier-Gall C, Ballester J, Schmitt-Kopplin P, et al. Impact of glutathione on wines oxidative stability: a combined sensory and metabolomic study. Front Chem. (2018) 6:182. doi: $10.3389 /$ fchem. 2018.00182 
12. Blasi F, Rocchetti G, Montesano D, Lucini L, Chiodelli G, Ghisoni S, et al. Changes in extra-virgin olive oil added with Lycium barbarum L. carotenoids during frying: chemical analyses and metabolomic approach. Food Res Int. (2018) 105:507-16. doi: 10.1016/j.foodres.2017.11.061

13. Gu EJ, Kim DW, Jang GJ, Song SH, Lee JI, Lee SB, et al. Mass-based metabolomic analysis of soybean sprouts during germination. Food Chem. (2017) 217:311-9. doi: 10.1016/j.foodchem.2016.08.113

14. Santos MCB, Lima LRD, Nascimento FR, do Nascimento TP, Cameron LC, Ferreira MSL. Metabolomic approach for characterization of phenolic compounds in different wheat genotypes during grain development. Food Res Int. (2019) 124:118-28. doi: 10.1016/j.foodres.2018.08.034

15. Yuan YF, Zhao YP, Yang JL, Jiang YM, Lu F, Jia YX, et al. Metabolomic analyses of banana during postharvest senescence by ${ }^{1} \mathrm{H}$-high resolution-NMR. Food Chem. (2017) 218:406-12. doi: 10.1016/j.foodchem.2016.09.080

16. Buchner N, Krumbein A, Rohn S, Kroh LW. Effect of thermal processing on the flavonols rutin and quercetin. Rapid Commun Mass Sp. (2006) 20:322935. doi: 10.1002/rcm.2720

17. Hou ZH, Qin PY, Zhang Y, Cui SH, Ren GX. Identification of anthocyanins isolated from black rice (Oryza sativa L.) and their degradation kinetics. Food Res Int. (2013) 50:691-7. doi: 10.1016/j.foodres.2011.07.037

18. Cevallos-Casals BA, Cisneros-Zevallos L. Stability of anthocyanin-based aqueous extracts of Andean purple corn and red-fleshed sweet potato compared to synthetic and natural colorants. Food Chem. (2004) 86:6977. doi: 10.1016/j.foodchem.2003.08.011

19. Ortu E, Caboni P. Levels of 5-hydroxymethylfurfural, furfural, 2-furoic acid in sapa syrup, Marsala wine and bakery products. Int J Food Prop. (2017) 20:S2543-51. doi: 10.1080/10942912.2017.1373668

20. Zhang YY, Song Y, Hu XS, Liao XJ, Ni YY, Li QH. Effects of sugars in batter formula and baking conditions on 5-hydroxymethylfurfural and furfural formation in sponge cake models. Food Res Int. (2012) 49:43945. doi: 10.1016/j.foodres.2012.07.012

21. Louarme L, Billaud C. Evaluation of ascorbic acid and sugar degradation products during fruit dessert processing under conventional or ohmic heating treatment. Lwt-Food Sci Technol. (2012) 49:184-7. doi: 10.1016/j.lwt.2011.12.035

22. Hou DX, Kai K, Li JJ, Lin SG, Terahara N, Wakamatsu M, et al. Anthocyanidins inhibit activator protein 1 activity and cell transformation: structure-activity relationship and molecular mechanisms. Carcinogenesis. (2003) 25:29-36. doi: 10.1093/carcin/bgg184

23. Hou DX, Yanagita T, Uto T, Masuzaki S, Fujii M. Anthocyanidins inhibit cyclooxygenase-2 expression in LPS-evoked macrophages: structure-activity relationship and molecular mechanisms involved. Biochem Pharmacol. (2005) 70:417-25. doi: 10.1016/j.bcp.2005.05.003

24. Tanaka T, Tanaka T, Tanaka M. Potential cancer chemopreventive activity of protocatechuic acid. Int J Clin Exp Med. (2011) 3:2733. doi: 10.1016/j.jecm.2010.12.005

25. Teegarden MD, Knobloch TJ, Weghorst CM, Cooperstone JL, Peterson DG. Storage conditions modulate the metabolomic profile of a black raspberry nectar with minimal impact on bioactivity. Food Funct. (2018) 9:4593601. doi: 10.1039/C8FO00639C

26. Hussain MI, Syed QA, Khattak MNK, Hafez B, Reigosa MJ, El-Keblawy A. Natural product coumarins: biological and pharmacological perspectives. Biologia. (2019) 74:863-88. doi: 10.2478/s11756-019-00242-x

27. Zhao MY, Luo YH, Li Y, Liu X, Wu JH, Liao XJ, et al. The identification of degradation products and degradation pathway of malvidin-3-glucoside and malvidin-3,5-diglucoside under microwave treatment. Food Chem. (2013) 141:3260-7. doi: 10.1016/j.foodchem.2013.05.147

28. Sadilova E, Stintzing FC, Carle R. Thermal degradation of acylated and non-acylated anthocyanins. J Food Sci. (2006) 71:C504-12. doi: 10.1111/j.1750-3841.2006.00148.x

29. Mancilla-Margalli NA, López MG. Generation of maillard compounds from inulin during the thermal processing of Agave tequilana Weber var. Azul J Agric Food Chem. (2002) 50:806-12. doi: 10.1021/jf01 10295
30. Lourenço A, Gominho J, Pereira H. Chemical characterization of lignocellulosic materials by analytical pyrolysis. In: P Kusch, editor. Analytical Pyrolysis. London, UK: IntechOpen Limited. (2018). p. 1-22. doi: 10.5772/intechopen.80556

31. Wong Z, Chen KF, Li J. Formation of vanillin and syringaldehyde in an oxygen delignification process. Bioresources. (2010) 5:1509-16.

32. Choi MY, Chai C, Park JH, Lim J, Lee J, Kwon SW. Effects of storage period and heat treatment on phenolic compound composition in dried Citrus peels (Chenpi) and discrimination of Chenpi with different storage periods through targeted metabolomic study using HPLC-DAD analysis. $J$ Pharmaceut Biomed. (2011) 54:638-45. doi: 10.1016/j.jpba.2010.09.036

33. Jeong SM, Kim SY, Kim DR, Jo SC, Nam KC, et al. Effect of heat treatment on the antioxidant activity of extracts from citrus peels. J Agric Food Chem. (2004) 52:3389-93. doi: 10.1021/jf049899k

34. Lou SN, Lin YS, Hsu YS, Chiu EM, Ho CT. Soluble and insoluble phenolic compounds and antioxidant activity of immature calamondin affected by solvents and heat treatment. Food Chem. (2014) 161:24653. doi: 10.1016/j.foodchem.2014.04.009

35. Rakwal R, Agrawal GK, Yonekura M, Kodama O. Naringenin 7-Omethyltransferase involved in the biosynthesis of the flavanone phytoalexin sakuranetin from rice (Oryza sativa L.). Plant Sci. (2000) 155:21321. doi: 10.1016/S0168-9452(00)00223-5

36. Katsumata S, Hamana K, Horie K, Toshima H, Hasegawa M. Identification of sternbin and naringenin as detoxified metabolites from the rice flavanone phytoalexin sakuranetin by Pyricularia oryzae. Chem Biodivers. (2017) 14:e1600240. doi: 10.1002/cbdv.201600240

37. Rinsant D, Chatel G, Jérôme F. Efficient and selective oxidation of D-glucose into gluconic acid under low-frequency ultrasonic irradiation. Chemcatchem. (2014) 6:3355-9. doi: 10.1002/cctc.201402604

38. Zhou P, Zhang J, Zhang Y, Liu Y, Liang J, Liu B, et al. Generation of hydrogen peroxide and hydroxyl radical resulting from oxygen-dependent oxidation of L-ascorbic acid via copper redox-catalyzed reactions. RSC Adv. (2016) 6:38541-7. doi: 10.1039/C6RA02843H

39. Yen GC, Lee TC. Mutagen formation in the reaction of maillard browning products, 2-acetylpyrrole and its analogues, with nitrite. Food Chem Toxicol. (1986) 24:1303-8. doi: 10.1016/0278-6915(86)90062-1

40. Zou TT, Kang L, Yang C, Song HL, Liu Y. Flavour precursor peptide from an enzymatic beef hydrolysate Maillard reaction-II: mechanism of the synthesis of flavour compounds from a sulphur-containing peptide through a Maillard reaction. Lwt-Food Sci Technol. (2019) 110:8-18. doi: 10.1016/j.lwt.2019.04.022

41. Fang ML, Ivanisevic J, Benton HP, Johnson CH, Patti GJ, Hoang LT, et al. Thermal degradation of small molecules: a global metabolomic investigation. Anal Chem. (2015) 87:10935-41. doi: 10.1021/acs.analchem.5b03003

42. Bitskinashvili K, Gabriadze I, Kutateladze T, Vishnepolsky B, Mikeladze D, Datukishvili N. Effects of thermal-acid treatment on degradation and amplification of wheat and maize DNA. J Food Nutr Res. (2018) 57:242-51.

43. Maga JA. Lactones in foods. Crit Rev Food Sci. (1976) 8:156. doi: 10.1080/10408397609527216

44. Wishart DS, Jewison T, Guo AC, Wilson M, Knox C, Liu YF, et al. HMDB 3.0-the human metabolome database in 2013. Nucl Acids Res. (2013) 41:8017. doi: $10.1093 /$ nar/gks1065

Conflict of Interest: The authors declare that the research was conducted in the absence of any commercial or financial relationships that could be construed as a potential conflict of interest.

Copyright (c) 2020 Zheng, Wu, Yang, Zhou, Hu, Ou and Liu. This is an open-access article distributed under the terms of the Creative Commons Attribution License (CC BY). The use, distribution or reproduction in other forums is permitted, provided the original author(s) and the copyright owner(s) are credited and that the original publication in this journal is cited, in accordance with accepted academic practice. No use, distribution or reproduction is permitted which does not comply with these terms. 